\title{
Search for a compressed supersymmetric spectrum with a light gravitino
}

\author{
Juhi Dutta, ${ }^{a}$ Partha Konar, ${ }^{b}$ Subhadeep Mondal, ${ }^{a}$ Biswarup Mukhopadhyaya ${ }^{a}$ \\ and Santosh Kumar Rai ${ }^{a}$ \\ ${ }^{a}$ Regional Centre for Accelerator-based Particle Physics, \\ Harish-Chandra Research Institute, HBNI, \\ Chhatnag Road, Jhusi, Allahabad 211019, India \\ ${ }^{b}$ Physical Research Laboratory, \\ Ahmedabad 380009, India \\ E-mail: juhidutta@hri.res.in, konar@prl.res.in, \\ subhadeepmondal@hri.res.in, biswarup@hri.res.in, skrai@hri.res.in
}

ABSTRACT: Presence of the light gravitino as dark matter candidate in a supersymmetric (SUSY) model opens up interesting collider signatures consisting of one or more hard photons together with multiple jets and missing transverse energy from the cascade decay. We investigate such signals at the $13 \mathrm{TeV}$ LHC in presence of compressed SUSY spectra, consistent with the Higgs mass as well as collider and dark matter constraints. We analyse and compare the discovery potential in different benchmark scenarios consisting of both compressed and uncompressed SUSY spectra, considering different levels of compression and intermediate decay modes. Our conclusion is that compressed spectra upto $2.5 \mathrm{TeV}$ are likely to be probed even before the high luminosity run of LHC. Kinematic variables are also suggested, which offer distinction between compressed and uncompressed spectra yielding similar event rates for photons + multi-jets $+\mathbb{E}_{T}$.

KeYwords: Supersymmetry Phenomenology

ARXIV EPRINT: 1704.04617 


\section{Contents}

1 Introduction 1

2 Compressed spectrum with a gravitino LSP 4

2.1 Relevant branching ratios 5

2.1.1 Variation of $\operatorname{BR}(\widetilde{g} \rightarrow g \widetilde{G}) \quad 5$

2.1.2 Variation of $\operatorname{BR}\left(\widetilde{q}_{L / R} \rightarrow q \widetilde{G}\right) \quad 6$

$\begin{array}{lll}\text { 2.1.3 Variation of } \operatorname{BR}\left(\widetilde{\chi}_{1}^{0} \rightarrow X \widetilde{G}\right) & 7\end{array}$

$\begin{array}{llr}3 & \text { Benchmark points } & 8\end{array}$

4 Collider analysis $\quad 10$

$\begin{array}{lll}4.1 & \text { Simulation set up and analysis } & 11\end{array}$

$\begin{array}{ll}4.2 & \text { Distinction of compressed and uncompressed spectra }\end{array}$

$\begin{array}{lll}4.3 \mathrm{eV} \text { gravitino } & 20\end{array}$

$\begin{array}{llr}5 & \text { Summary and conclusion } & 21\end{array}$

\section{Introduction}

The Large Hadron Collider (LHC) has already accumulated a substantial volume of data with $\sqrt{s}=13 \mathrm{TeV}$. Although the discovery of a scalar resembling the Higgs boson [1-6] in the Standard Model (SM) has laid the foundation of a success story, the absence of any new physics signal is a source of exasperation to those in search of physics beyond the SM (BSM). This applies to the search for phenomenologically viable supersymmetric (SUSY) scenarios as well. The non-observation of any supersymmetric particle so far at the LHC has strengthened the limits on many such low scale SUSY models. While the large production cross-section of the coloured SUSY particles (sparticles) are already pushing the existing mass limits to the $2 \mathrm{TeV}$ mark with the initial data at the $13 \mathrm{TeV}$ run, the weakly interacting sparticles are still not that severely constrained [7, 8]. With the LHC already operating close to its near maximum centre-of-mass energy, consistent improvements in luminosity is expected to help accumulate enough data which will help probe the coloured sector mass to almost $3 \mathrm{TeV}$ with some improvements for the weakly interacting sector too.

This lack of evidence for any low scale SUSY events prompted the idea of a compressed sparticle spectrum [9-21], where the lightest SUSY particle (LSP) and the heavier sparticle states may be nearly degenerate. In such realizations of the mass spectra, the resulting final state jets and leptons from the decay cascades of the parent particles are expected to be very soft, including the overall missing transverse energy which is a manifestation of the available visible transverse momenta. As events with such soft final states would be 
susceptible to low acceptance efficiencies in the detectors and therefore lead to much smaller event rates in the conventional SUSY search channels. In the absence of hard leptons or jets arising from the cascade, one has to rely on tagging the jets or photons originating from the initial state radiation (ISR) or final state radiation (FSR) to detect such events where the available missing transverse momenta is characterized by the stability of the LSP in the cascades. Usually, in most SUSY models, the lightest neutralino $\left(\widetilde{\chi}_{1}^{0}\right)$ is assumed to be the LSP. Thus, such signals allow a much lighter SUSY spectrum compared to the conventional channels with hard leptons, jets and large missing transverse momentum [22-32].

However, in the presence of a light gravitino $(\widetilde{G})$ in the spectrum, such as in gauge mediated SUSY breaking (GMSB) models [33-40], the $\widetilde{\chi}_{1}^{0}$ is quite often the next-to-lightest SUSY particle (NLSP), which decays into a $\widetilde{G}$ and a gauge/Higgs boson. Search strategy for such scenarios, therefore, is expected to be significantly different. In this case, one would always expect to find one or more hard leptons/jets/photons in the final state originating from the $\widetilde{\chi}_{1}^{0}$ decay, irrespective of whether the SUSY mass spectrum is compressed or not. Hence detecting events characterizing such a signal is expected to be much easier, with the preferred channel being the photon mode. Given the fact that the hard photon(s) can easily be tagged for these events in a relatively compressed spectrum of the SUSY particles with the NLSP, one need not rely on the radiated jets for signal identification, thereby improving the cut efficiency significantly. If one considers a fixed gravitino mass, the photon(s) originating from the $\widetilde{\chi}_{1}^{0}$ decay will be harder as $m_{\widetilde{\chi}_{1}^{0}}$ becomes heavier. Hence these hard photon associated signals can be very effective to probe a heavy SUSY spectrum with a light gravitino as there would rarely be any SM events with such hard photons in the final state.

While the light gravitino scenario yields large transverse missing energy $\left(\mathbb{F}_{T}\right)$ as well as hard photon(s) and jet(s), the question remains as to whether its presence obliterate the information on whether the MSSM part of the spectrum is compressed or not. In this work, we have demonstrated how such information can be extracted. Our study in this direction contains the following new observations:

- A set of kinematic observables are identified involving hardness of the photon(s), the transverse momenta $\left(p_{T}\right)$ of the leading jets and also the $\mathbb{F}_{T}$, which clearly brings out the distinction between a compressed and an uncompressed spectrum with similar signal rates. We have studied different benchmarks with varied degree of compression in the spectrum in this context.

- The characteristic rates of the $n-\gamma$ (where $n \geq 1$ ) final state in a compressed spectrum scenario have been obtained and the underlying physics has been discussed.

- The circumstances under which, for example, a gluino in a compressed MSSM spectrum prefers to decay into a gluon and a gravitino rather than into jets and a neutralino have been identified. In this context, we have also found some remarkable effects of a eV-scale gravitino though such a particle can not explain the cold dark matter (DM) content of the universe. 
The experimental collaborations have considered light gravitino scenarios and derived bounds on the coloured sparticles [41-48]. The ATLAS collaboration recently published their analysis on a SUSY scenario with a light $\widetilde{G}$ with the $13 \mathrm{TeV}$ data accumulated at an integrated luminosity of $13.3 \mathrm{fb}^{-1}$ [48]. In this analysis, $\widetilde{\chi}_{1}^{0}$ is considered to be a binohiggsino mixed state decaying into $\gamma \widetilde{G}$ and(or) $Z \widetilde{G}$ resulting in the final state " $n_{1} \gamma+n_{2}$ jets $+\not_{T}$ " where $n_{1} \geq 1$ and $n_{2}>2$. The $13 \mathrm{TeV}$ data puts a stringent constraint on the sparticle masses excluding $m_{\widetilde{g}}$ upto $1950 \mathrm{GeV}$ subject to the lightest neutralino mass close to $1800 \mathrm{GeV}$ [46-48], which is a significant improvement on the bounds obtained after the $8 \mathrm{TeV}$ run with $20.3 \mathrm{fb}^{-1}$ integrated luminosity [42, 44]. We note that, in order to derive the limits from the collider data, the experimental collaboration considers signal events coming from gluino pair production only, while assuming the rest of the coloured sparticles viz. squarks to be much heavier to contribute to the signal. The robustness of the signal however does not differentiate whether such a heavy SUSY spectrum (leaving aside the gravitino) are closely spaced in mass or have a widely split mass spectrum, and whether it is just a single sparticle state that contributes to the signal or otherwise. We intend to impress through this work that such a signal would also be able to distinguish such alternate possibilities quite efficiently.

In an earlier work while assuming a similar compression in the sparticle spectrum [18] we had shown that in order to get a truly compressed ${ }^{1}$ pMSSM spectrum consistent with a $125 \mathrm{GeV}$ Higgs boson and the flavour and dark matter (DM) constraints, one has to have the $\widetilde{\chi}_{1}^{0}$ mass at or above $2 \mathrm{TeV}$ with the entire coloured sector lying slightly above. Such a spectrum is now seemingly of interest given the present experimental bounds obtained in $\widetilde{G}$ LSP scenario. ${ }^{2}$ In this work, we aim to extend our previous study by adding to the spectrum, a $\widetilde{G}$ LSP with mass, at most, in the eV-keV range. Rest of the pMSSM spectrum lies above the $\mathrm{TeV}$ range to be consistent with the experimental bounds. This is in contrast to existing studies done earlier for gravitino LSP which we compare by studying the prospects of uncompressed spectra having relatively larger mass gaps between the coloured sparticles and $\widetilde{\chi}_{1}^{0}$, but with event rates similar to that of the compressed spectra. Since the kinematics of the decay products in the two cases are expected to be significantly different, we present some kinematical variables which clearly distinguish a compressed spectrum from an uncompressed one, in spite of comparable signal rates in both cases.

The paper is organised in the following way. In section 2 we discuss about the phenomenological aspects of a SUSY spectrum with gravitino LSP and then move on to study the variation of the branching ratios of squark, gluino and the lightest neutralino into gravitino associated and other relevant decay modes. In section 3 we present some sample benchmark points representative of our region of interest consisting of both compressed and uncompressed spectra that are consistent with the existing constraints. Subsequently, in section 4 we proceed to our collider analysis with these benchmark points and present the details of our simulation and obtained results. Finally, in section 5 we summarise our results and conclude.

\footnotetext{
${ }^{1}$ Mass gap between the heaviest coloured sparticle and the LSP neutralino has to be around $100 \mathrm{GeV}$.

${ }^{2}$ Note that the bounds on the squark-gluino masses in the compressed region with $\widetilde{\chi}_{1}^{0}$ LSP are still much weaker. In such cases, the gluinos and first two generation squarks are excluded upto $650 \mathrm{GeV}$ and $450 \mathrm{GeV}$ respectively [30].
} 


\section{Compressed spectrum with a gravitino LSP}

The NLSP decaying into a gravitino and jets/leptons/photons give rise to very distinct signals at the LHC. Both the ATLAS and CMS collaborations have studied these signal regions for a hint of GMSB-like scenarios [41-48]. Note that, a pure GMSB like scenario is now under tension after the discovery of the $125 \mathrm{GeV}$ Higgs boson [49-51]. It is very difficult to fit a light Higgs boson within this minimal framework, mostly because of small mixing in the scalar sector. As a consequence, the stop masses need to be pushed to several $\mathrm{TeV}$ in order to obtain the correct Higgs mass, thus rendering such scenarios uninteresting in the context of LHC. However, some variations of the pure GMSB scenario are capable of solving the Higgs mass issue and can still give visible signals within the LHC energy range $[52,53]$. Since we are only interested in the phenomenology of these models here, a detailed discussion on their theoretical aspects is beyond the scope of this paper.

Although the lightest neutralino $\left(\widetilde{\chi}_{1}^{0}\right)$ is the more popular DM candidate in SUSY theories, gravitino $(\widetilde{G})$ as the LSP has its own distinct phenomenology. The $\widetilde{G}$ is directly related to the effect of SUSY breaking via gauge mediation and all its couplings are inversely proportional to the Planck mass $\left(\sim 10^{18} \mathrm{GeV}\right)$ and thus considerably suppressed. The hierarchy of the sparticle masses depend on the SUSY breaking mechanism and can result in $\widetilde{G}$ getting mass which is heavier, comparable or lighter than the other superpartners. Thus if it happens to be the LSP in the theory, $\widetilde{G}$ can also be a good DM candidate [54-59] making such scenarios of considerable interest in the context of the LHC. In addition, having $\widetilde{G}$ as a DM candidate also relaxes the DM constraints on the rest of the SUSY spectrum by a great deal, allowing them to be very heavy while being consistent with a light $\widetilde{G}$ DM. However, a very light $\widetilde{G}$ is mostly considered to be warm DM. Present cosmological observations require a light gravitino to have a mass close to a few $\mathrm{keV}[60,61]$ at least, if it has to explain the cold DM relic density. However, the kinematic characteristics of events when the NLSP decays into a gravitino are mostly independent of whether the gravitino is in the $\mathrm{keV}$ range or even lower in mass. Some special situations where the difference is of some consequence have been discussed in section 4.3. Of course, the presence of a gravitino much lighter than a $\mathrm{keV}$ will require the presence of some additional cold DM candidate.

Note that with $\widetilde{G}$ as the LSP decay branching ratios (BR) of the sparticles can be significantly modified since they can now decay directly into $\widetilde{G}$ instead of decaying into $\widetilde{\chi}_{1}^{0}$, which may significantly alter their collider signals. The decay width $(\Gamma)$ of a sparticle, $\operatorname{scalar}(\widetilde{f})$ or gaugino $(\widetilde{V})$, decaying into their respective SM counterparts, chiral fermion $(f)$ or gauge $\operatorname{boson}(V)$, and $\widetilde{G}$ is given by $[62]$

$$
\begin{aligned}
\Gamma(\tilde{f} \rightarrow f \widetilde{G}) & =\frac{1}{48 \pi} \frac{m_{\widetilde{f}}^{5}}{M_{P l}^{2} m_{\widetilde{G}}^{2}}\left[1-\left(\frac{m_{\widetilde{G}}}{m_{\widetilde{f}}}\right)^{2}\right]^{2} \\
\Gamma(\widetilde{V} \rightarrow V \widetilde{G}) & =\frac{1}{48 \pi} \frac{m_{\widetilde{V}}^{5}}{M_{P l}^{2} m_{\widetilde{G}}^{2}}\left[1-\left(\frac{m_{\widetilde{G}}}{m_{\widetilde{V}}}\right)^{2}\right]^{3}
\end{aligned}
$$

where $M_{P l}$ is the Planck scale. Thus it is evident that this decay mode starts to dominate once the sparticles become very heavy and the $\widetilde{G}$ becomes light. 


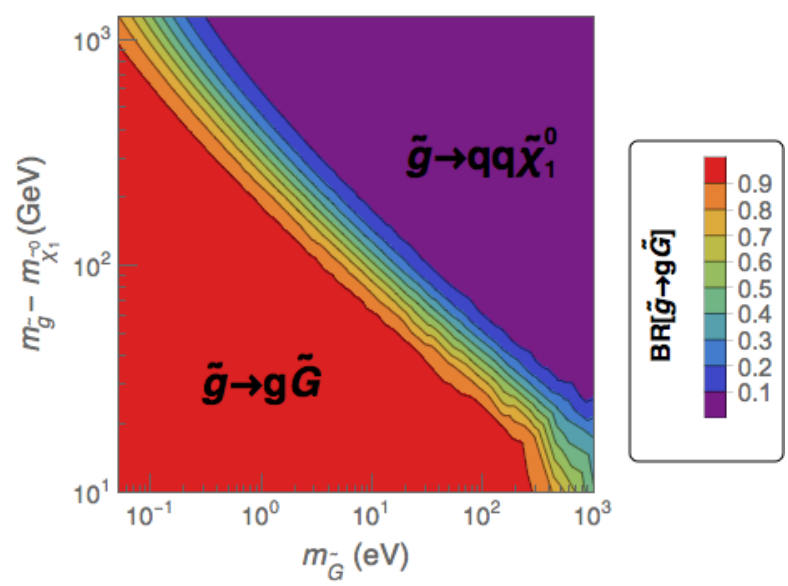

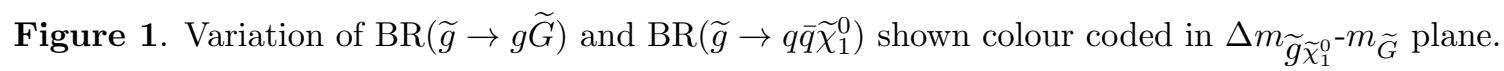

\subsection{Relevant branching ratios}

In this section, we discuss the variation of the branching ratios (BR) of various sparticles into the LSP gravitino. Since in this analysis we aim to study the production of the coloured sparticles and their subsequent decays into the $\widetilde{G}$ via $\widetilde{\chi}_{1}^{0}$, the decay modes of $\widetilde{g}$, $\widetilde{q}$ and $\widetilde{\chi}_{1}^{0}$ are of our primary interest. While considering the decay modes, we focus on a simplified assumption that the decaying coloured sparticle is the next-to-next-lightest supersymmetric particle (NNLSP) with $\widetilde{\chi}_{1}^{0}$ as the NLSP and $\widetilde{G}$ as the LSP. The BR computation and spectrum generation was done using SPheno [63-65] for a phenomenological MSSM (pMSSM) like scenario with one additional parameter, i.e, the gravitino mass $\left(m_{\widetilde{G}}\right)$.

\subsubsection{Variation of $\mathbf{B R}(\widetilde{g} \rightarrow g \widetilde{G})$}

In figure 1 we show the variation of two relevant gluino decay mode channels viz. $\widetilde{g} \rightarrow g \widetilde{G}$ and $\widetilde{g} \rightarrow q \bar{q} \widetilde{\chi}_{1}^{0}$ where all the squarks are heavier, as a function of $\Delta m_{\widetilde{g}_{\widetilde{\chi}_{1}^{0}}}=m_{\widetilde{g}}-m_{\widetilde{\chi}_{1}^{0}}$ and $m_{\widetilde{G}}$. The gluino mass has been fixed to $m_{\widetilde{g}}=2500 \mathrm{GeV}$ while $m_{\widetilde{\chi}_{1}^{0}}$ has been varied such that $\Delta m_{\widetilde{g}_{\widetilde{\chi}_{1}^{0}}}$ varies within $10-1500 \mathrm{GeV}$. Note that the $\widetilde{\chi}_{1}^{0}$ is considered to be dominantly binolike. In the absence of its two-body decay mode into squark-quark pairs, the gluino can only decay via $\widetilde{g} \rightarrow g \widetilde{G}$ or $\widetilde{g} \rightarrow q \bar{q} \widetilde{\chi}_{1}^{0}$. The other two-body decay mode $\widetilde{g} \rightarrow g \widetilde{\chi}_{1}^{0}$ being loop suppressed, remains mostly subdominant compared to these two decay modes. Hence, only the two relevant channels are shown in the figure. Note that, $\operatorname{BR}\left(\widetilde{g} \rightarrow q \bar{q} \widetilde{\chi}_{1}^{0}\right)$ includes the sum of all the off-shell contributions obtained from the first two generation squarks which in this case lie about $100 \mathrm{GeV}$ above $m_{\widetilde{g}}$. As the gravitino mass gets heavier, $\operatorname{BR}(\widetilde{g} \rightarrow g \widetilde{G})$ decreases since, the corresponding partial width is proportional to the inverse square of $m_{\widetilde{G}}$. Similarly, as $m_{\widetilde{\chi}_{1}^{0}}$ keeps increasing, $\operatorname{BR}\left(\widetilde{g} \rightarrow q \bar{q} \widetilde{\chi}_{1}^{0}\right)$ goes on decreasing. Note that, the BR for the 3-body decay mode can decrease further with increase in the corresponding squark masses. However, even for a $\mathrm{keV} \widetilde{G}, \operatorname{BR}(\widetilde{g} \rightarrow g \widetilde{G})$ can remain significantly large provided there is sufficient compression in the mass gap $\left(\Delta m_{\widetilde{g}_{\widetilde{\chi}_{1}^{0}}} \sim 10 \mathrm{GeV}\right)$ as seen in figure 1. 

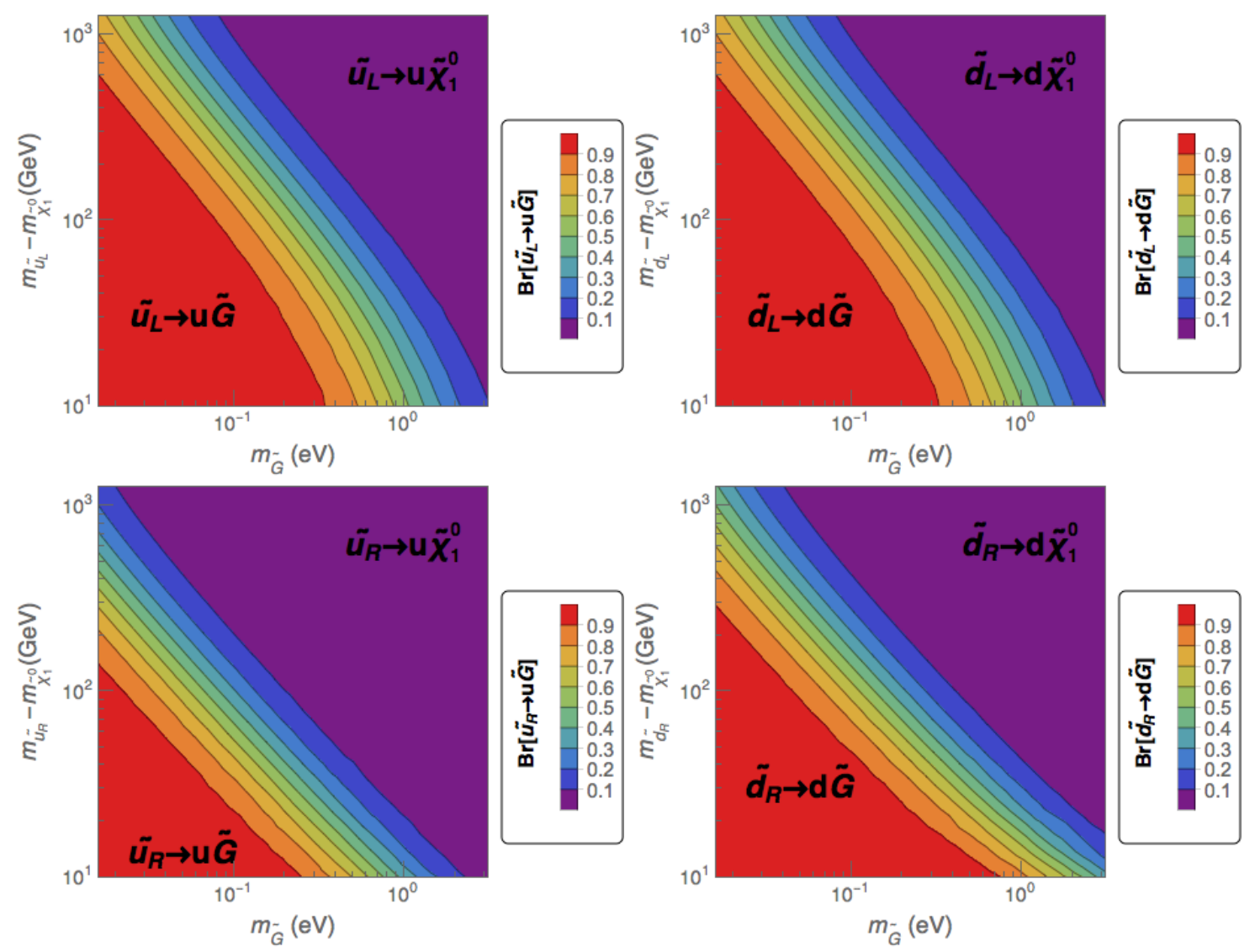

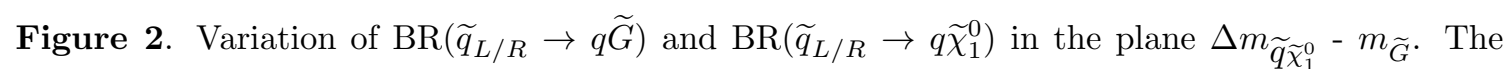
plots on the left show the distributions corresponding to the up-squarks and the plots on the right show the same for the down-squarks.

\subsubsection{Variation of $\operatorname{BR}\left(\widetilde{q}_{L / R} \rightarrow q \widetilde{G}\right)$}

Next we look into the relevant decay modes of the first two generation squarks ${ }^{3}$ when they are the NNLSP's. In this case, we assume that the gluino is heavier than the squarks, so that the dominant two-body decay modes available to the squarks are $\widetilde{q}_{L / R} \rightarrow q \widetilde{G}$ and $\widetilde{q}_{L / R} \rightarrow q \widetilde{\chi}_{1}^{0}$. Unlike the previous case, here the gravitino decay branching ratio has competition from another two-body decay mode. Although the decay into $\widetilde{G}$ does not depend on the $\mathrm{L}$ and R-type of the squarks, $\operatorname{BR}\left(\widetilde{q}_{L / R} \rightarrow q \widetilde{\chi}_{1}^{0}\right)$ is expected to be different depending on the composition of the $\widetilde{\chi}_{1}^{0}$. For simplicity, we choose the $\widetilde{\chi}_{1}^{0}$ to be purely bino-like as before. The squark masses are fixed at $m_{\widetilde{q}}=2500 \mathrm{GeV}$ and the NLSP mass, $m_{\widetilde{\chi}_{1}^{0}}$ is varied as before such that $\Delta m_{\widetilde{q}_{1}^{0}}=m_{\widetilde{q}}-m_{\widetilde{\chi}_{1}^{0}}$ varies in a wide range, $10-1500 \mathrm{GeV}$. The branching probabilities are shown in figure 2 where the plots on the left (right) shows the decay branching ratios of $u_{L / R}\left(d_{L / R}\right)$. As the coupling of $\widetilde{q}_{L}$ with the SM-quark and bino-component of $\widetilde{\chi}_{1}^{0}$ is proportional to $\sqrt{2} g \tan \theta_{W}\left(I_{3 q}-e_{q}\right)$ while that of $\widetilde{q}_{R}$ is

\footnotetext{
${ }^{3}$ Since the production cross-section of the third generation squarks are substantially smaller than those of the first two generations, we do not consider the production of the stop and sbottom states. Hence we only discuss the decays of $\tilde{u}_{L / R}$ and $\tilde{d}_{L / R}$.
} 
proportional to $\sqrt{2} g \tan \theta_{W} e_{q}$, where $g, e_{q}$ and $I_{3 q}$ represents $\mathrm{SU}(2)$ gauge coupling, electric charge of the SM-quark and its isospin respectively [62], we find a noticeable variation in decay probabilities of $\widetilde{q}_{L}$ and $\widetilde{q}_{R}$ for the same choice of mass spectrum. This implies that the right-handed squarks couple more strongly with the $\widetilde{\chi}_{1}^{0}$ compared to the lefthanded ones. As a result, although the partial decay widths of the squarks decaying into gravitino and quarks are identical for squarks of similar mass, the corresponding BR vary slightly depending on their handedness. This feature is evident in figure 2. The coupling strength of $\widetilde{u}_{R}$ with $\widetilde{\chi}_{1}^{0}$ is larger by a factor of four compared to that of $\widetilde{u}_{L}$. The same coupling corresponding to $\widetilde{d}_{R}$ is larger by a factor of two compared to that of $\widetilde{d}_{L}$. Hence the difference in the BR distributions is more manifest for the up-type squarks. The magnitude of the coupling strengths corresponding to $\widetilde{u}_{L}$ and $\widetilde{d}_{L}$ are exactly same and hence we have obtained similar distributions corresponding to those.

The BR distributions indicate that as we go on compressing the SUSY spectrum, the gravitino decay mode becomes more and more relevant but only if its mass is around or below the eV range. We, therefore, conclude that for a $\mathrm{keV} \widetilde{G}$, the decay mode $\widetilde{g} \rightarrow g \widetilde{G}$ may be of importance but only for the cases where the gluino mass lies very close to the NLSP neutralino mass. For the first two generation squarks and a $\mathrm{keV} \widetilde{G}$, the $\operatorname{BR}\left(\widetilde{q}_{L / R} \rightarrow q \widetilde{G}\right)$ is very small and the decay of the squarks into $\widetilde{\chi}_{1}^{0}$ dominates in the absence of a lighter gluino. As evident, the gravitino decay mode can be of significance for LHC studies if $m_{\widetilde{G}} \sim \mathrm{eV}$. However, such a light $\widetilde{G}$ is strongly disfavoured from DM constraints as mentioned before.

\subsubsection{Variation of $\operatorname{BR}\left(\widetilde{\chi}_{1}^{0} \rightarrow X \widetilde{G}\right)$}

The last two subsections point out the situations where the NLSP can be bypassed in the decay of strongly interacting superparticles. Such events tend to reduce the multiplicity of hard photons in SUSY-driven final states. In contrast, in the case where the SUSY cascades lead to a $\widetilde{\chi}_{1}^{0}$ NLSP, the $\widetilde{\chi}_{1}^{0}$ may further decay into gravitino along with a $Z, \gamma$ or the Higgs boson $(h)$ depending upon its composition. ${ }^{4}$ The $h$-associated decay width is entirely dependent on the higgsino component of $\widetilde{\chi}_{1}^{0}$ while $\Gamma\left(\widetilde{\chi}_{1}^{0} \rightarrow \gamma \widetilde{G}\right)$ depends entirely on the bino and wino component of $\widetilde{\chi}_{1}^{0}$ whereas the $Z$-associated decay width has a partial dependence on all the components that make up the $\widetilde{\chi}_{1}^{0}$. The functional dependence on the different composition strengths of $\widetilde{\chi}_{1}^{0}$ in its decay width can be summarised as [62]:

$$
\begin{aligned}
& \Gamma\left(\widetilde{\chi}_{1}^{0} \rightarrow \gamma \widetilde{G}\right) \propto\left|N_{11} \cos \theta_{W}+N_{12} \sin \theta_{W}\right|^{2} \\
& \Gamma\left(\widetilde{\chi}_{1}^{0} \rightarrow Z \widetilde{G}\right) \propto\left(\left|N_{11} \sin \theta_{W}-N_{12} \cos \theta_{W}\right|^{2}+\frac{1}{2}\left|N_{14} \cos \beta-N_{13} \sin \beta\right|^{2}\right) \\
& \Gamma\left(\widetilde{\chi}_{1}^{0} \rightarrow h \widetilde{G}\right) \propto\left|N_{14} \sin \alpha-N_{13} \cos \alpha\right|^{2}
\end{aligned}
$$

where, $N_{i j}$ are the elements of the neutralino mixing matrix, $\theta_{W}$ is the Weinberg mixing angle, $\alpha$ is the neutral Higgs mixing angle and $\beta$ corresponds to the ratio of the up and down type Higgs vacuum expectation values (VEVs). Note that the partial decay widths are proportional to $m_{\widetilde{\chi}_{1}^{0}}^{5} /\left(M_{P l}^{2} m_{\widetilde{G}}^{2}\right)$ and hence if $m_{\widetilde{G}}$ is too large, the total decay width

\footnotetext{
${ }^{4}$ In principle, $\widetilde{\chi}_{1}^{0}$ may decay into the other neutral Higgs states also which we assume to be heavier.
} 

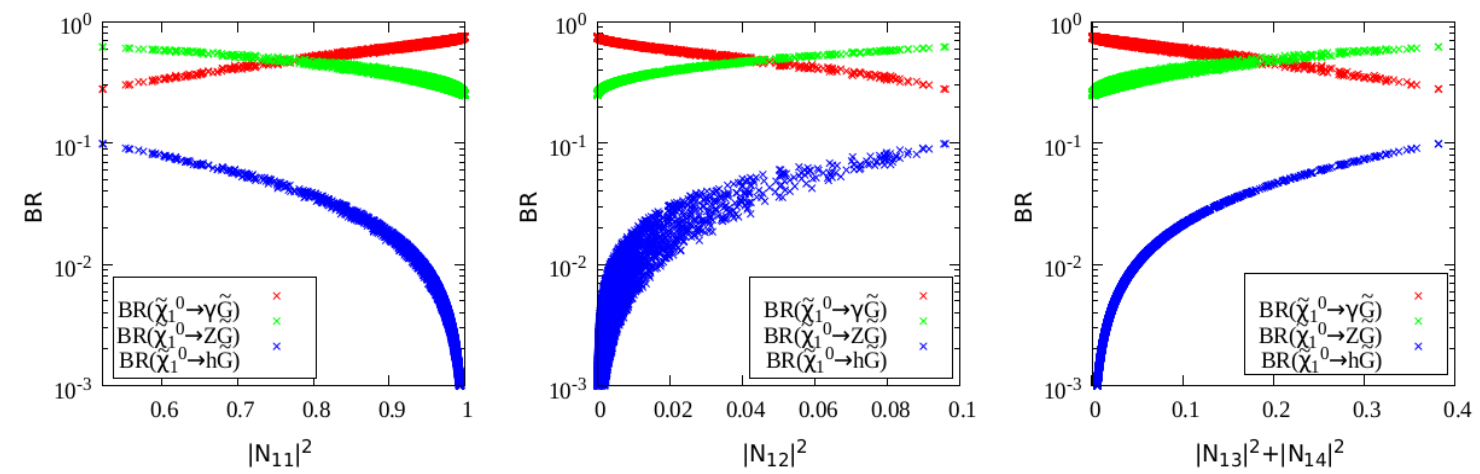

Figure 3. Vatiation of the three relevant BRs of $\widetilde{\chi}_{1}^{0}$ decay modes with its bino, wino and higgsino components. The red, green and blue lines correspond to $\operatorname{BR}\left(\widetilde{\chi}_{1}^{0} \rightarrow \gamma \widetilde{G}\right), \operatorname{BR}\left(\widetilde{\chi}_{1}^{0} \rightarrow Z \widetilde{G}\right)$ and $\mathrm{BR}\left(\widetilde{\chi}_{1}^{0} \rightarrow h \widetilde{G}\right)$ respectively.

of $\widetilde{\chi}_{1}^{0}$ may become too small such that it will not decay within the detector. Although the decay width is also dependent upon $m_{\widetilde{\chi}_{1}^{0}}$, one finds that for a $2500 \mathrm{GeV} \widetilde{\chi}_{1}^{0}$, and a $\mathrm{MeV} \widetilde{G}$ the neutralino becomes long-lived. In figure 3 we show the variation of the three relevant BRs with the composition of the $\widetilde{\chi}_{1}^{0}$. Here we have varied $M_{1}, M_{2}$ and $\mu$ in the range $[2: 2.5] \mathrm{TeV}$ with the condition $\mu>M_{2}>M_{1}$ such that $\widetilde{\chi}_{1}^{0}$ is bino-like most of the time with different admixtures of wino and higgsino components. The other relevant mixing parameter $\tan \beta$ is kept fixed at 10 . The red, green and blue colours correspond to $\operatorname{BR}\left(\widetilde{\chi}_{1}^{0} \rightarrow \gamma \widetilde{G}\right), \operatorname{BR}\left(\widetilde{\chi}_{1}^{0} \rightarrow Z \widetilde{G}\right)$ and $\operatorname{BR}\left(\widetilde{\chi}_{1}^{0} \rightarrow h \widetilde{G}\right)$ respectively. $\left|N_{11}\right|^{2}$ indicates the binofraction in the composition of $\widetilde{\chi}_{1}^{0}$. Similarly, $\left|N_{12}\right|^{2}$ and $\left|N_{13}^{2}\right|+\left|N_{14}\right|^{2}$ represent the wino and higgsino components respectively. As can be clearly seen from the plots, obtaining $100 \% \operatorname{BR}\left(\widetilde{\chi}_{1}^{0} \rightarrow \gamma \widetilde{G}\right)$ is not possible even if the bino and(or) wino components are close to 1 , since the $Z$-mode is always present. However, the $h$-associated decay channel can be easily suppressed with a relatively larger $\mu$. Motivated by this behaviour of the BRs, we choose to work with a signal consisting of at least one photon for our collider analysis. In our case, the $\widetilde{\chi}_{1}^{0}$ being dominantly bino-like, it decays mostly into a $\gamma$ and a $\widetilde{G}$. However, the $Z \widetilde{G}$ decay mode has a substantial BR $(\sim 25 \%)$. The higgsino admixture in $\widetilde{\chi}_{1}^{0}$ being small, the $h \widetilde{G}$ decay mode is not considered in this work. However it is worth noting that this particular channel can be the dominant mode for a higgsino-dominated NLSP and could also be an interesting mode of study, which we leave for future work.

\section{Benchmark points}

For our analysis we choose a few benchmark points that would represent the salient features of a compressed sparticle spectrum with varying compression strengths while also categorically defining a few points that are more in line with current SUSY searches with $\widetilde{G}$ LSP by the CMS and ATLAS collaborations at the LHC. We insure that our benchmark choices are consistent with all existing experimental constraints. We consider both compressed and uncompressed spectra, with bino-like $\widetilde{\chi}_{1}^{0}$ as the NLSP and a keV gravitino as the LSP and warm dark matter candidate. For one of the benchmarks, we also show 


\begin{tabular}{|c|c|c|c|c|c|c|c|c|}
\cline { 2 - 9 } \multicolumn{1}{c|}{} & \multicolumn{7}{c|}{ Compressed spectra } & \multicolumn{2}{c|}{ Uncompressed spectra } \\
\hline Parameters & C1 & C2 & C3 & C4 & C5 & C6 & U1 & U2 \\
\hline$M_{1}$ & 2623 & 2451 & 2671 & 2608 & 2550 & 2486 & 704 & 1200 \\
$M_{2}$ & 2710 & 2610 & 2710 & 2710 & 2610 & 2610 & 2310 & 2310 \\
$M_{3}$ & 2480 & 2280 & 2560 & 2601 & 2380 & 2285 & 1747 & 1747 \\
$A_{t}$ & 2895 & 2895 & -3295 & -3750 & -3197 & -2895 & 2895 & 2895 \\
$\mu$ & 4000 & 4000 & 4000 & 4000 & 3500 & 4000 & 3000 & 3000 \\
$\tan \beta$ & 15 & 15 & 9 & 6 & 25 & 15 & 15 & 15 \\
$M_{A}$ & 2500 & 2500 & 1800 & 1800 & 2500 & 2500 & 2500 & 2500 \\
\hline$m_{\widetilde{g}}$ & 2678 & 2456 & 2746 & 2783 & 2562 & 2468 & 2102 & 2102 \\
$m_{\widetilde{q}_{L}}$ & 2729 & 2468 & 2734 & 2753 & 2571 & 2467 & 4721 & 4721 \\
$m_{\widetilde{q}_{R}}$ & 2727 & 2466 & 2730 & 2751 & 2574 & 2468 & 4742 & 4742 \\
$m_{\widetilde{t}_{1}}$ & 2707 & 2457 & 2652 & 2625 & 2532 & 2543 & 4680 & 4678 \\
$m_{\widetilde{t}_{2}}$ & 2837 & 2593 & 2857 & 2863 & 2718 & 2725 & 4767 & 4765 \\
$m_{\widetilde{b}_{1}}$ & 2787 & 2501 & 2782 & 2778 & 2594 & 2598 & 4560 & 4558 \\
$m_{\widetilde{b}_{2}}$ & 2846 & 2570 & 2846 & 2846 & 2677 & 2669 & 4746 & 4744 \\
$m_{\widetilde{\ell}_{L}}$ & 2703 & 2452 & 2703 & 2703 & 2572 & 2503 & 4335 & 4336 \\
$m_{\widetilde{\ell}_{R}}$ & 2700 & 2455 & 2700 & 2700 & 2585 & 2495 & 4365 & 4366 \\
$m_{\widetilde{\tau}_{1}}$ & 2706 & 2443 & 2707 & 2709 & 2600 & 2576 & 4332 & 4332 \\
$m_{\widetilde{\tau}_{2}}$ & 2882 & 2514 & 2882 & 2881 & 2671 & 2622 & 4375 & 4375 \\
$m_{\widetilde{\nu}_{L}}$ & 2701 & 2450 & 2701 & 2701 & 2570 & 2501 & 4335 & 4335 \\
$m_{\widetilde{\chi}_{1}^{0}}$ & 2600 & 2428 & 2646 & 2585 & 2526 & 2462 & 699 & 1191 \\
$m_{\widetilde{\chi}_{2}^{0}}$ & 2726 & 2614 & 2724 & 2724 & 2619 & 2617 & 2383 & 2383 \\
$m_{\widetilde{\chi}_{1}^{ \pm}}$ & 2726 & 2614 & 2725 & 2724 & 2619 & 2617 & 2382 & 2382 \\
\hline$m_{h}$ & 123 & 123 & 124 & 124 & 125 & 124 & 125 & 125 \\
$\Delta M_{i}$ & 129 & 40 & 100 & 198 & 48 & 6 & 1403 & 911 \\
\hline & & & & & & & & \\
\hline
\end{tabular}

Table 1. Low energy input parameters and the relevant sparticle masses, (in GeV), for the compressed $\left(\mathbf{C}_{i}, \mathrm{i}=1, \ldots, 6\right)$ and uncompressed $(\mathbf{U} 1, \mathbf{U} 2)$ benchmarks. Here, $\Delta M_{i}=m_{i}-m_{\widetilde{\chi}_{1}^{0}}$ where $m_{i}$ represents the mass of the heaviest coloured sparticle $\left(\widetilde{g} / \widetilde{q}_{k},(\mathrm{k}=1,2)\right)$ and $m_{\widetilde{\chi}_{1}^{0}}$, the mass of the NLSP. For all benchmarks, the gravitino mass, $m_{\widetilde{G}}=1 \mathrm{keV}$.

the effect of an eV mass gravitino LSP. The final benchmarks used in this study are shown in table 1.

The mass spectrum and decays of the sparticles are computed using SPheno-v3.3.6 [6365]. We restrict the light CP-even Higgs mass to be in the range $122-128 \mathrm{GeV}$, i.e, within $3-\sigma$ range of the measured Higgs mass $[1-4]$ and including theoretical uncertainty of $4 \mathrm{GeV}$. Note that when the mass spectrum is compressed, all squark/gluino (which are nearly degenerate in mass) production channels contribute significantly to the signal. For all the benchmark points, the squarks and gluino decay directly or via cascades to the binolike $\widetilde{\chi}_{1}^{0}$ NLSP. The $\widetilde{\chi}_{1}^{0}$ then dominantly decays to a photon and gravitino and, to a lesser 
extent, a $Z$ boson and gravitino. This leads to either a mono-photon or a diphoton signal with jets and $\mathscr{H}_{T}$ which defines our signal. To evade constraints from photon(s) searches at the LHC for simplified models [41-48], we require the sparticles in a compressed spectrum such as ours, to be much heavier than the existing experimental limits. We have checked this for our spectra represented by the benchmark points, with the NLSP mass lying in the range $2.4-2.6 \mathrm{TeV}$ with varied masses and hierarchy of the coloured sparticles with respect to the NLSP. Amongst them, C6 is the utmost compressed spectra, with a mass gap, $\Delta M_{i}$ $\sim 6 \mathrm{GeV}$ between the coloured sparticles and the NLSP of mass $2462 \mathrm{GeV}$, followed by C2, C5 where the mass gap is in the range of $40-50 \mathrm{GeV}$ and the NLSP masses are 2428 and $2526 \mathrm{GeV}$ respectively. We have also considered benchmarks C1, C3 and C4 such that the mass gap between the coloured sparticles and NLSP are slightly higher and lie in the range of $100-200 \mathrm{GeV}$.

We also choose various possible mass hierarchical arrangements of the squarks and gluino to accommodate different cascades contributing to the signal. For example, C1 and C3 have different squark-gluino mass hierarchical stuctures in the strong sector. This leads to different jet distributions in the two cases. C2 and C5, on the other hand, are similar in the arrangement of the sparticles, however placed within $50 \mathrm{GeV}$ from the NLSP, which represents a much more compressed scenario. Finally we consider two uncompressed spectra U1, U2 with NLSP mass $\sim 700 \mathrm{GeV}$ and $\sim 1200 \mathrm{GeV}$ and gluinos with mass $\sim 1.4 \mathrm{TeV}$ and $\sim 1 \mathrm{TeV}$ above the NLSP respectively. Since the photons arise from the NLSP decays, a heavier NLSP gives rise to a harder photon, having better chances of passing the analysis cuts. Thus the difference in the signal cross-sections differ on account of the difference in hardness of the photons and the resulting cut efficiencies in these two cases.

Benchmark points $\mathbf{U 1}, \mathbf{U} \mathbf{2}$ are in fact replications of the simplified scenarios that are considered by experimental collaborations to put limits on SUSY particle masses. For both these benchmark points, we have kept the squarks very heavy $(\sim 4-5 \mathrm{TeV})$ so that the gluino pair production is the only dominant contributing channel. However, we have only focussed on uncompressed spectra with event rates comparable to those of the compressed spectra. Since the large mass gap between the gluino and NLSP allow for multiple hard jets to be produced as opposed to the compressed case, we further exploit this feature to differentiate compressed from uncompressed scenarios with comparable event rates during signal analysis.

\section{Collider analysis}

We look for multi-jet signals associated with very hard photon(s) and missing transverse energy $\left(\mathscr{F}_{T}\right)$ in the context of SUSY with gravitino as the LSP. For such GMSB kind of models with a keV gravitino, a very clear signature arises from the decay of the NLSP neutralino into a photon and a gravitino. If the NLSP-LSP mass difference is large enough, two hard photons would appear in the final state at the end of a SUSY cascade. The lightest neutralino, if bino-like, decays dominantly into a photon and gravitino $(\sim 75 \%)$ while a small fraction decays into $Z$ boson and gravitino $(\sim 25 \%)$. For cases with $\widetilde{\chi}_{1}^{0}$ having a significant 
higgsino component, we get comparable branching fractions for its decay into $Z$ boson or a Higgs boson, besides photons, along with $\widetilde{G}$. For simplicity, we have considered a bino-like $\widetilde{\chi}_{1}^{0}$ as the NLSP. Note that the signal strength consisting of very hard photons in the final state can be affected by the composition of the NLSP as we have discussed before. The $\widetilde{\chi}_{1}^{0}$ decay into a $Z \widetilde{G}$ however still remains relevant for the bino-like $\widetilde{\chi}_{1}^{0}$ and as a result, gives rise to a monophoton signal at the LHC along with the diphoton channel, associated with large missing transverse energy. The existing LHC constraints in such scenarios have already pushed the $\widetilde{\chi}_{1}^{0}-\widetilde{q}-\widetilde{g}$ mass bounds above $1.5 \mathrm{TeV}$ which automatically result in a large $\widetilde{\chi}_{1}^{0}-\widetilde{G}$ mass gap. This gives rise to very high $p_{T}$ photons in the final states, which are very easy to detect and also highly effective to suppress the SM background events.

In this work, we consider six benchmark points for compressed spectra (C1 - C6) such that the entire coloured sector (apart from $\widetilde{t}_{2}$ and $\widetilde{b}_{2}$ ) lie within $200 \mathrm{GeV}$ of the $\widetilde{\chi}_{1}^{0}$ $\left(\mathrm{m}_{\chi_{1}^{0}} \sim 2.4-2.6 \mathrm{TeV}\right)$. We then estimate signal rates of final state events with at least one or more hard photons arising from all possible squark-gluino pair production modes. We also study a couple of uncompressed spectra $(\mathbf{U 1 , U 2 )}$ such that both the compressed and uncompressed spectra produce similar event rates for our signal. In these spectra, the NLSP mass is around 700 and $1200 \mathrm{GeV}$ respectively and the gluino is the lightest coloured sparticle having a large $(\sim 1-1.4 \mathrm{TeV})$ mass gap with the NLSP. The squarks are chosen to be heavier (4-5 TeV) and are essentially decoupled from rest of the spectrum. The large mass gap between the NLSP and the coloured sector ensures multiple hard jets from their decay cascades besides the hard photons. Thus with different mass gaps and squark-gluino hierarchy among the compressed and uncompressed spectra, the jet profiles are expected to be significantly different for the benchmark points. Following the existing ATLAS analysis [48], which provides the most stringent constraint on the SUSY spectrum with a light gravitino LSP, we determine the signal event rates for our choice of benchmark points. Since we have also chosen compressed and uncompressed spectra such that the final state event rates are equal or comparable after analysis, it is a priori difficult to determine which scenario such a signal reflects. Keeping this in mind, we propose a set of kinematic variables, besides the usual kinematic ones like $\mathbb{F}_{T}$ and $M_{E f f}$, which highlight the distinctive features of compression in a SUSY spectra over an uncompressed one with $\widetilde{G}$ as the LSP, although both have comparable signal rates.

\subsection{Simulation set up and analysis}

We consider the pair production and associated production processes of all coloured sparticles at $\sqrt{s}=13 \mathrm{TeV}$ LHC. Parton level events are generated using Madgraph5 (v2.2.3) [66, 67 for the following processes with upto two extra partons at the matrix element level:

$$
p p \rightarrow \widetilde{q}^{*} \widetilde{q}, \widetilde{q} \widetilde{g}, \widetilde{q} \widetilde{q}, \widetilde{q}^{*} \widetilde{q}^{*}, \widetilde{q}^{*} \widetilde{g}, \widetilde{g} \widetilde{g}
$$

We reject any intermediate resonances at the matrix element level, which may arise in the decay cascades of the sparticles from two or more different processes, to avoid double counting of Feynman diagrams to the processes. The parton level events are then showered using Pythia (v6) [68]. To correctly model the hard ISR jets and reduce double counting of 
jets coming from the showers as well as the matrix element partons, MLM matching [69, 70] of the shower jets and the matrix element jets have been performed using the shower- $\mathrm{k}_{T}$ algorithm with $\mathrm{p}_{T}$ ordered showers by choosing a matching scale (QCUT) $120 \mathrm{GeV}$ [71]. The default dynamic factorisation and renormalization scales [72] have been used in Madgraph whereas the PDF chosen is CTEQ6L [73]. After the showering, hadronisation and fragmentation effects performed by Pythia, subsequent detector simulation of the hadron level events are carried out by the fast simulator Delphes-v3.3.3 [74-76]. The jets are reconstructed using Fastjet [77] with a minimum $p_{T}$ of $20 \mathrm{GeV}$ in a cone of $\Delta R=0.4$ using the anti- $k_{t}$ algorithm [78]. The charged leptons $(e, \mu)$ are reconstructed in a cone of $\Delta R=0.2$ with the maximum amount of energy deposit allowed in the cone limited to $10 \%$ of the $p_{T}$ of the lepton. Photons are reconstructed in a cone of $\Delta R=0.4$, with the maximum energy deposit in the cone as per ATLAS selection criteria [48].

For background estimation, we focus on the most dominant SM backgrounds for photon $(\mathrm{s})+$ jets $+\mathbb{E}_{T}$ signal at $13 \mathrm{TeV}$ LHC, such as: $\gamma+\leq 4$ jets, $\gamma \gamma+\leq 3$ jets, $W \gamma+\leq 3$ jets, $Z \gamma+\leq 3$ jets and $t \bar{t} \gamma+$ jets. The sort of extremely hard $p_{T}$ photons that we expect in our signal events, are unlikely to be present in SM processes in abundance and the hard photons will arise mostly from the tails of the $p_{T}^{\gamma}$ distributions. Hence in order to obtain a statistically exhaustive event sample, we choose a hard $p_{T_{\gamma}}>200 \mathrm{GeV}$ cut as a preselection for the parton level events for the leading photon while generating the background events. For MLM matching of the jets, the matching scale was chosen in the range $30-50 \mathrm{GeV}$ as applicable for electroweak SM processes.

Some other SM processes, such as QCD, $t \bar{t}+$ jets, $W+$ jets, $Z+$ jets, in spite of having no direct sources of hard photons, may also contribute to the background owing to their large production cross-sections coupled with mistagging of jets or leptons leading to fake photons. However, the cumulative effect of hard $p_{T}^{\gamma}$ as well as $\mathbb{F}_{T}$ and $M_{E f f}$ requirement renders these contributions negligible.

Primary event selection criteria. We identify the charged leptons $(e, \mu)$, photons and jets as per the following selection criteria (A0) for signal and background events alike:

- Leptons $(\ell=e, \mu)$ are selected with $p_{T}^{\ell}>25 \mathrm{GeV},\left|\eta^{e}\right|<2.37$ and $\left|\eta^{\mu}\right|<2.70$ and excluding the transitional pseudorapidity window $1.37<\left|\eta^{\ell}\right|<1.52$ between the ECAL barrel and end cap of the calorimeter.

- Photons are identified with $p_{T}^{\gamma}>75 \mathrm{GeV}$ and $\left|\eta^{\gamma}\right|<2.47$ excluding $1.37<\left|\eta^{\gamma}\right|<1.52$.

- Reconstructed jets have $p_{T}^{j}>30 \mathrm{GeV}$ and lie within $\left|\eta^{j}\right|<2.5$.

- All reconstructed jets have a large azimuthal separation with $\overrightarrow{\mathscr{E}}_{T}$, given by $\Delta \phi\left(\right.$ jet, $\left.\vec{E}_{T}\right)>0.4$ to reduce fake contributions to missing transverse energy arising from hadronic energy mismeasurements.

- The jets are separated from other jets by $\Delta R_{j j}>0.4$ and from the reconstructed photons by $\Delta R_{\gamma j}>0.4$. 


\begin{tabular}{|c|c|c|c|c|c|c|c|}
\hline \multicolumn{3}{|c|}{ Signal } & \multicolumn{5}{|c|}{ Effective cross-section (in fb) after the cuts } \\
\hline $\begin{array}{c}\text { Benchmark } \\
\text { Points }\end{array}$ & $\begin{array}{c}\text { Production } \\
\text { cross-section(fb) }\end{array}$ & A0 + A1 & A2 & A3 & A4 & A5 & A6 \\
\hline C1 & 0.26 & 0.22 & 0.18 & 0.14 & 0.14 & 0.13 & 0.12 \\
C2 & 0.80 & 0.68 & 0.37 & 0.30 & 0.30 & 0.28 & 0.26 \\
C3 & 0.23 & 0.18 & 0.10 & 0.08 & 0.08 & 0.08 & 0.08 \\
C4 & 0.21 & 0.15 & 0.12 & 0.08 & 0.08 & 0.08 & 0.07 \\
C5 & 0.49 & 0.34 & 0.15 & 0.13 & 0.13 & 0.12 & 0.11 \\
C6 & 0.77 & 0.61 & 0.13 & 0.11 & 0.11 & 0.10 & 0.09 \\
U1 & 0.20 & 0.10 & 0.09 & 0.08 & 0.07 & 0.05 & 0.05 \\
U2 & 0.20 & 0.13 & 0.12 & 0.10 & 0.09 & 0.08 & 0.08 \\
\hline
\end{tabular}

Table 2. Signal Cross-sections (NLO+NLL) for all the benchmark points listed in table 1 corresponding to $\left(\geq 1 \gamma+>2\right.$ jets $\left.+\mathbb{F}_{T}\right)$ final state. For all the points, $m_{\widetilde{G}}=1 \mathrm{keV}$.

With these choices of final state selection criteria we now proceed to select the events for our analysis.

Signal region: $\geq \mathbf{1} \gamma+>\mathbf{2}$ jets $+\boldsymbol{F}_{\boldsymbol{T}}$. We look into final states with at least 1 photon, multiple jets and large $\mathbb{E}_{T}$. Amongst the existing analyses for the same final state carried by the experimental collaborations, the ATLAS analysis imposes a more stringent constraint on the new physics parameter space and hence we have implemented the same set of cuts as enlisted below for our analysis:

- A1: the final state events comprise of at least one photon and the leading photon $\left(\gamma_{1}\right)$ must have $p_{T}^{\gamma_{1}}>400 \mathrm{GeV}$.

- A2: there should be no charged leptons in the final state $\left(N_{\ell}=0\right)$ but at least 2 hard jets $\left(N_{j}>2\right)$.

- A3: the leading and sub-leading jets must be well separated from $\vec{f}_{T}$, such that $\Delta \phi\left(j, \overrightarrow{F_{T}}\right)>0.4$.

- A4: the leading photon must also be well separated from $\overrightarrow{\mathscr{H}_{T}}$ with $\Delta \phi\left(\gamma_{1}, \overrightarrow{F_{T}}\right)>0.4$.

- A5: as the light gravitinos would carry away a large missing transverse momenta, we demand that $\mathscr{F}_{T}>400 \mathrm{GeV}$.

- A6: we further demand effective mass, $M_{E f f}>2000 \mathrm{GeV}$, with $M_{E f f}=H_{T}+G_{T}+$ $\mathbb{F}_{T}$, where $H_{T}=\Sigma_{i} p_{T}\left(j_{i}\right)$ is the scalar sum of $p_{T}$ of all jets and $G_{T}=\Sigma_{j} p_{T}\left(\gamma_{j}\right)$ is the scalar sum of $p_{T}$ of all photons in the event.

In table 2 we have summarised the effect of the cuts A0-A6 for our signal on the respective benchmark points. All the production cross-sections in the table is scaled using NLO+NLL K-factors obtained from NLL_Fast [79-83].

As evident from table 2, cut efficiencies vary depending on the compression in the spectra. For example, the jet requirement affects the signal cross-section of $\mathbf{C 6}$ the most, 


\begin{tabular}{|c|c|c|}
\hline \multirow[t]{2}{*}{ Signal } & \multicolumn{2}{|c|}{ Luminosity $\mathcal{L}\left(\right.$ in $\left.\mathrm{fb}^{-1}\right)$ for } \\
\hline & $\mathcal{S}=3 \sigma$ & $\mathcal{S}=5 \sigma$ \\
\hline C1 & 68 & 189 \\
\hline $\mathrm{C} 2^{5}$ & 19 & 52 \\
\hline C3 & 139 & 385 \\
\hline $\mathrm{C} 4$ & 176 & 489 \\
\hline $\mathrm{C} 5$ & 79 & 219 \\
\hline C6 & 112 & 312 \\
\hline U1 & 326 & 904 \\
\hline U2 & 139 & 385 \\
\hline
\end{tabular}

Table 3. Required luminosity $(\mathcal{L})$ to obtain $3 \sigma$ and $5 \sigma$ statistical Significance $(\mathcal{S})$ of the signal at the $13 \mathrm{TeV}$ run of the LHC corresponding to the benchmark points.

since it is the most compressed spectra among all. Naturally, one would expect jet multiplicity to be smaller in this case compared to the others. As a result, the requirement $N_{j}>2$ reduces the corresponding signal cross-section by a significant amount, whereas, for the uncompressed spectra, U1 and U2, this cut has no bearing. The hard photon(s) in the signal events and the presence of direct source of $\mathbb{E}_{T}$ ensure that the $\mathbb{E}_{T}$ and $M_{E f f}$ cuts are easily satisfied by the selected events.

For the corresponding background events, we use the observed number of background events at ATLAS, which is 1, for the same final state studied at an integrated luminosity of $13.3 \mathrm{fb}^{-1}$ at $13 \mathrm{TeV}$ [48]. The statistical signal significance is computed using

$$
\mathcal{S}=\sqrt{2\left[(s+b) \ln \left(1+\frac{s}{b}\right)-s\right]}
$$

where $s$ and $b$ represent the remaining number of signal and background events after implementing all the cuts. In table 3 , we have shown the required integrated luminosity to obtain a $3 \sigma$ and $5 \sigma$ statistical significance for our signal corresponding to all the benchmark points.

The required luminosity for $3 \sigma$ and $5 \sigma$ statistical significance varies depending on the relative compression and heaviness of the spectra. As evident, C2 has the best discovery prospects and is likely to be probed very soon. C6 on the other hand, despite of having a similar squark-gluon spectra and a very similar production cross-section to that of C2, requires a much larger luminosity $\left(\sim 112 \mathrm{fb}^{-1}\right)$ to be probed. This is because the high amount of compression in the spectra reduces the cut efficiency significantly due to the jet multiplicity requirement. The required integrated luminosity for C1 and C5 is very similar although C5 has a relatively lighter coloured sector and thus a larger production cross-section compared to $\mathbf{C 1}$, as can be seen from table 2. However, the photon and jet selection criteria reduces the $\mathbf{C 5}$ cross-section making it comparable to that of $\mathbf{C 1}$. The situation is different for $\mathbf{U} \mathbf{1}$ which despite of having the lightest gluino, requires the largest

\footnotetext{
${ }^{5}$ On the face of it, this benchmark may be ruled out by the current searches at LHC. However, this is to be taken with some caution, since the search criteria suggested by us are slightly different from the ones used in the current experimental searches.
} 
luminosity $\left(\sim 326 \mathrm{fb}^{-1}\right)$ among all the benchmark points in order to be probed. The reason is two-fold. Firstly, the production cross-section in this case (and also for U2) is comprised of just the gluino-pair since the squarks are far too heavy to contribute. Secondly, the $\widetilde{\chi}_{1}^{0}$ being $\sim 700 \mathrm{GeV}$, the photons arising from $\widetilde{\chi}_{1}^{0}$ decay are relatively on the softer side and hence the photon selection criteria further reduces the signal cross-section. A similar squark-gluon spectra in presence of a heavier $\widetilde{\chi}_{1}^{0}$ (U2) therefore is likely to be probed with a much smaller luminosity $\left(\sim 139 \mathrm{fb}^{-1}\right)$ than $\mathbf{U} 1$. Thus it is evident from table 2 and 3, that given the present experimental constraints, a compressed spectra, unless it is too highly compressed such that the cut efficiency is reduced significantly, can improve the squark-gluino mass limits by a significant amount. For example, C2 can be probed with slightly little more luminosity than $13.3 \mathrm{fb}^{-1}$ but with a coloured spectra that lies in the vicinity of $2.5 \mathrm{TeV}$. This clearly suggests that a compressed spectra becomes much more quickly disfavoured over an uncompressed spectra with a gravitino LSP contrary to the case where a compressed SUSY spectrum appears as a saviour of low mass SUSY with a neutralino LSP. This is because of the hard photons that themselves act as a clear criterion to distinguish the signal over the SM background.

\subsection{Distinction of compressed and uncompressed spectra}

Given the inclusive hard photon $+\mathbb{F}_{T}$ signals, supposedly due to a light gravitino, can one ascertain whether the MSSM part of the spectrum is compressed or uncompressed? With this question in view, it is worthwhile to compare signals of both types with various degree of compression in presence of a light $(\sim \mathrm{keV})$ gravitino as the LSP. We show that the kind of compressed spectra we have used enhances the existing exclusion limit on the coloured sparticles. We consider different squark-gluino mass hierarchy represented by our choice of some sample benchmark points presented in table 1 . The $\widetilde{G}$ being almost massless in comparison to the $\widetilde{\chi}_{1}^{0}$ in consideration, the photons generated from the $\widetilde{\chi}_{1}^{0}$ decay into $\widetilde{G}$ are always expected to be very hard for both the compressed and uncompressed scenarios. This feature can be used to enhance the significance of the signal irrespective of the associated jets in the event. We provide a framework where one can use the properties of these jets in a novel way to distinguish between the two different scenarios in consideration even if they produce a similar event rate at the LHC. For illustration, let us consider the benchmark points, C5, C4 and U2 all of which result in nearly identical event rates for our signal and thus it is difficult to identify whether it is a signature of a compressed or an uncompressed spectra. It would be nice to have some kinematic variables which could be used to distinguish among the different kind of spectra. Subsequently, we have proposed few such variables which show distinctive features in their distributions depending on the relative hardness and multiplicity of the final state photon(s) and jets.

An uncompressed spectrum, such as $\mathbf{U} \mathbf{2}$ is characterized by a large mass gap between the strong sector sparticles and the NLSP $\left(\widetilde{\chi}_{1}^{0}\right)$. This ensures a large number of high $p_{T}$ jets from the cascades as compared to $\mathbf{C 5}$ and $\mathbf{C 4}$. The difference in jet multiplicity in the two cases is clearly visible in figure 4 where we have presented both the jet and photon multiplicity distributions for some sample compressed and uncompressed spectra. 

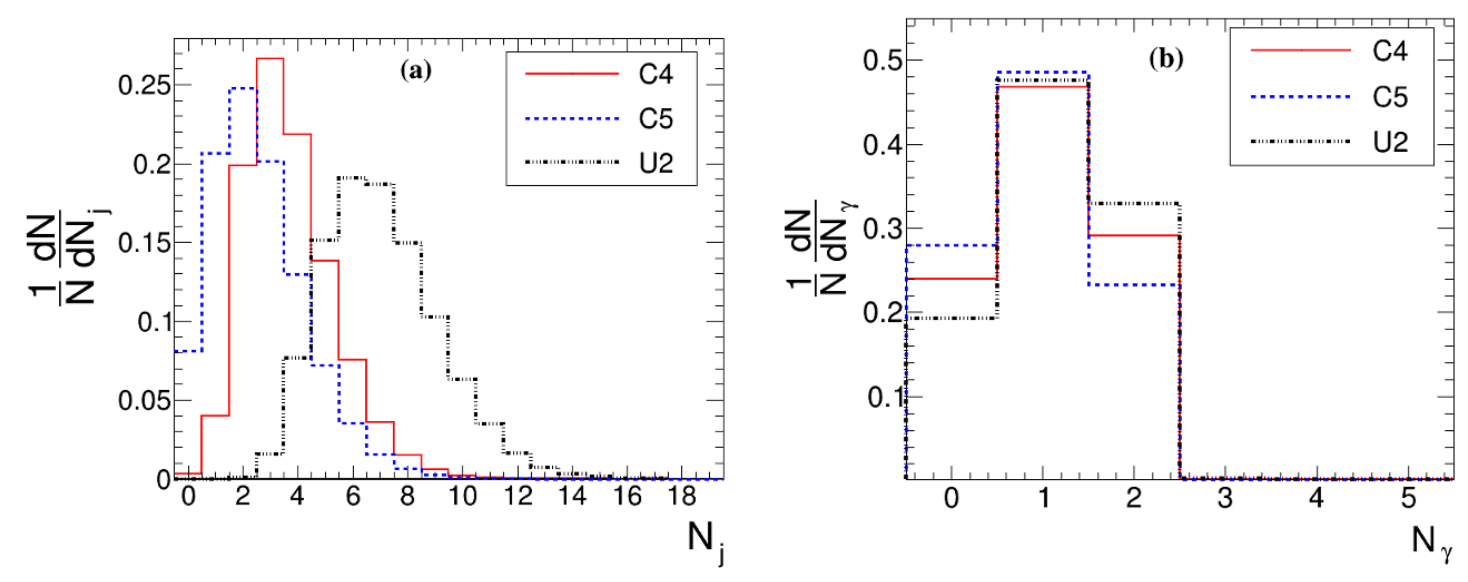

Figure 4. Normalized distributions for jet and photon multiplicity for the benchmark points C4, C5 and U2 representing moderately compressed, highly compressed and uncompressed scenarios respectively. Figure (a) has been prepared after implementing the selection cuts $\mathbf{A} \mathbf{0}+\mathbf{A} \mathbf{1}$ and figure (b) after A0.

The hard photons in the event are originated from the $\widetilde{\chi}_{1}^{0}$ decay and since for all our benchmark points the $\widetilde{\chi}_{1}^{0}$ is sufficiently heavy, the photon multiplicity peaks at a similar region for both the compressed and uncompressed spectra. However, the jets in the case of $\mathbf{U} 2$ are generated from the three body decay of the gluino into a pair of quarks and $\widetilde{\chi}_{1}^{0}$. As evident from figure 1, for the choices of the sparticle masses of $\mathbf{U} \mathbf{2}$, the other decay mode is highly suppressed. Hence one would naturally expect to obtain a large number of jets in the final state as shown in figure 4. C5 having a high degree of compression $\left(\Delta M_{i}=48 \mathrm{GeV}\right)$ in the parameter space results in least number of jets in the final state. C4, on the other hand, has a more relaxed compression $\left(\Delta M_{i}=198 \mathrm{GeV}\right)$ that gives rise to slightly harder cascade jets passing through the jet selection criteria resulting in a harder distribution than $\mathbf{C 5}$.

The relative difference in the compression factor $\left(\Delta M_{i}\right)$ among the three benchmark points are also visible in the jet $p_{T}$ distributions shown in figure 5 . As expected, the leading (figure 5(a)) and subleading (figure $5(\mathrm{~b})$ ) jet $p_{T}$ distributions predominantly show a harder peak for $\mathbf{U} 2$ as compared to C4, C5. However, hard jets may also arise from the $\widetilde{\chi}_{1}^{0}$ decaying to a $Z$ boson and gravitino $(\mathrm{BR} \sim 25 \%$ ) as the $Z$ decays dominantly into two jets. The $Z$ boson is expected to be highly boosted and thus one can easily obtain additional hard jets from its decay. These jets populate a small fraction of the total number of events and thus for a compressed spectra one of these jets can turn out to be the hardest jet in the event. This feature can be observed by the subdominant peak at $\sim 1000 \mathrm{GeV}$ for the leading jet $p_{T}$ distribution in figure 5 .

Figure 5(c) and (d) show the leading and subleading photon $p_{T}$ distributions respectively for C4, C5 and U2. The $\widetilde{\chi}_{1}^{0}$ mass in C4, C5 being $\sim 2.5 \mathrm{TeV}$, the photons produced from their decay are much harder than the leading jets in the spectra as opposed to the uncompressed spectra (U2) and hence, the peak in the photon $p_{T}$ distribution is signif- 

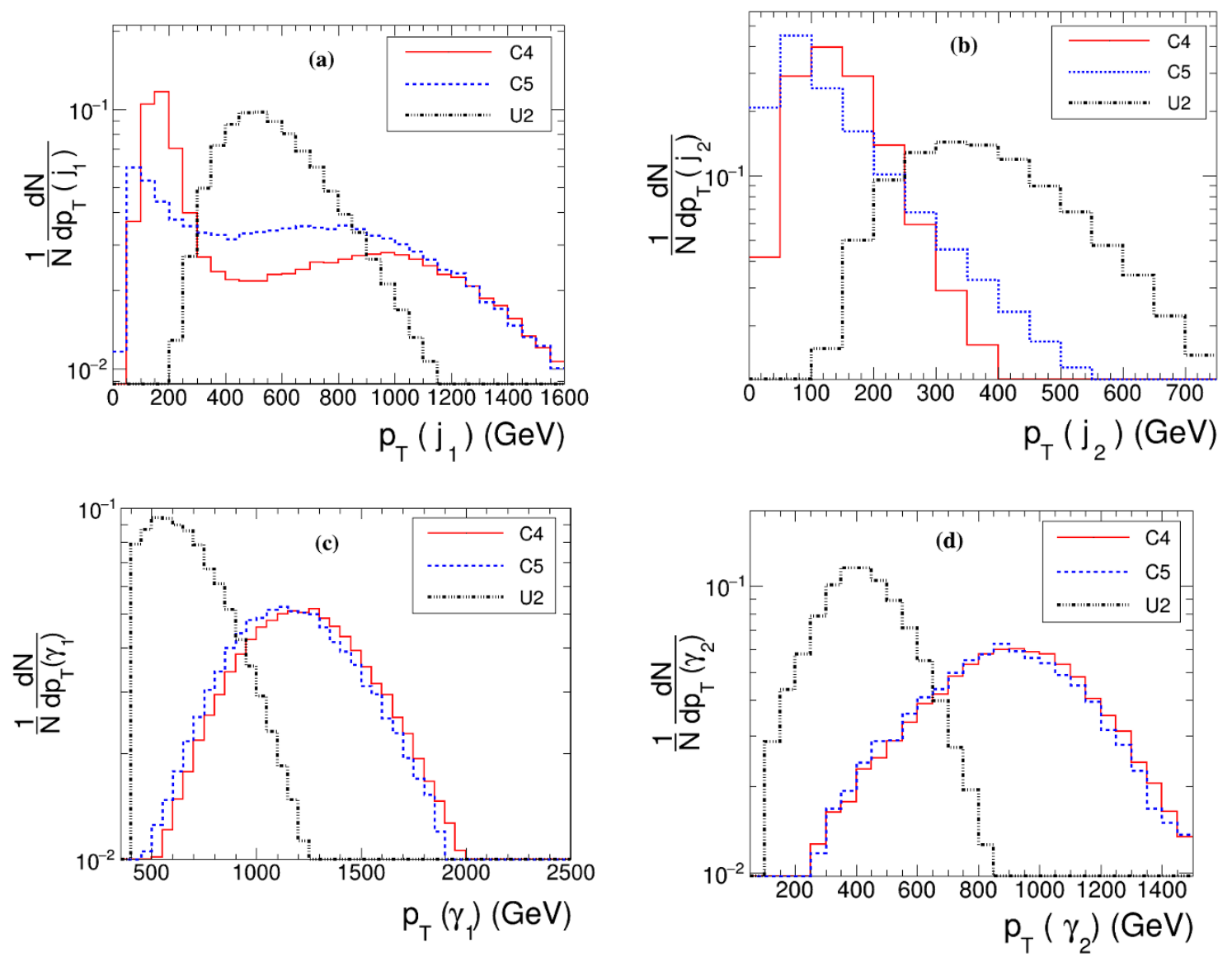

Figure 5. The leading and subleading jet and photon $p_{T}$ distributions for some of the benchmark points representing various compressed (C4), more compressed (C5) and uncompressed (U2) spectra after implementing the selection and analysis cuts A0-A6.

icantly shifted to lower values. Thus while the total hadronic energy, $H_{T}$ (figure 6(a)) peaks at a higher value for the uncompressed case owing to a large number of hard jets, $G_{T}$ (figure 6(b)) which is the scalar sum of all photon $p_{T}$, peaks at a lower value for the uncompressed case than the compressed cases.

Among other kinematic variables, one can also look into the $\mathbb{F}_{T}$ and $\mathrm{M}_{E f f}$ distributions to distinguish the compressed and uncompressed scenarios as shown in figure 6(c) and (d) respectively. Since the photons are almost always harder for the compressed spectra compared to the uncompressed cases, we have observed that the $\mathbb{E}_{T}$, required to balance the total visible transverse energy, is much harder for the former. Effective mass, $\mathrm{M}_{E f f}$ defined as the sum of $H_{T}, G_{T}$ and $\mathscr{F}_{T}$, also shows some small difference in the peak value for both cases. In U2, $G_{T}$ and $\mathbb{E}_{T}$ are softer than that for C4, C5 but $H_{T}$ is much harder resulting in the $M_{E f f}$ peaking at similar values for the both cases. However, since the photons are considerably harder than the jets in all cases, the effect being more pronounced for the compressed over the uncompressed case, the $\mathrm{M}_{E f f}$ distribution falls faster for U2 than $\mathbf{C 4}$ and $\mathbf{C 5}$ as can be seen from figure 6(b) and 6(d) respectively. 

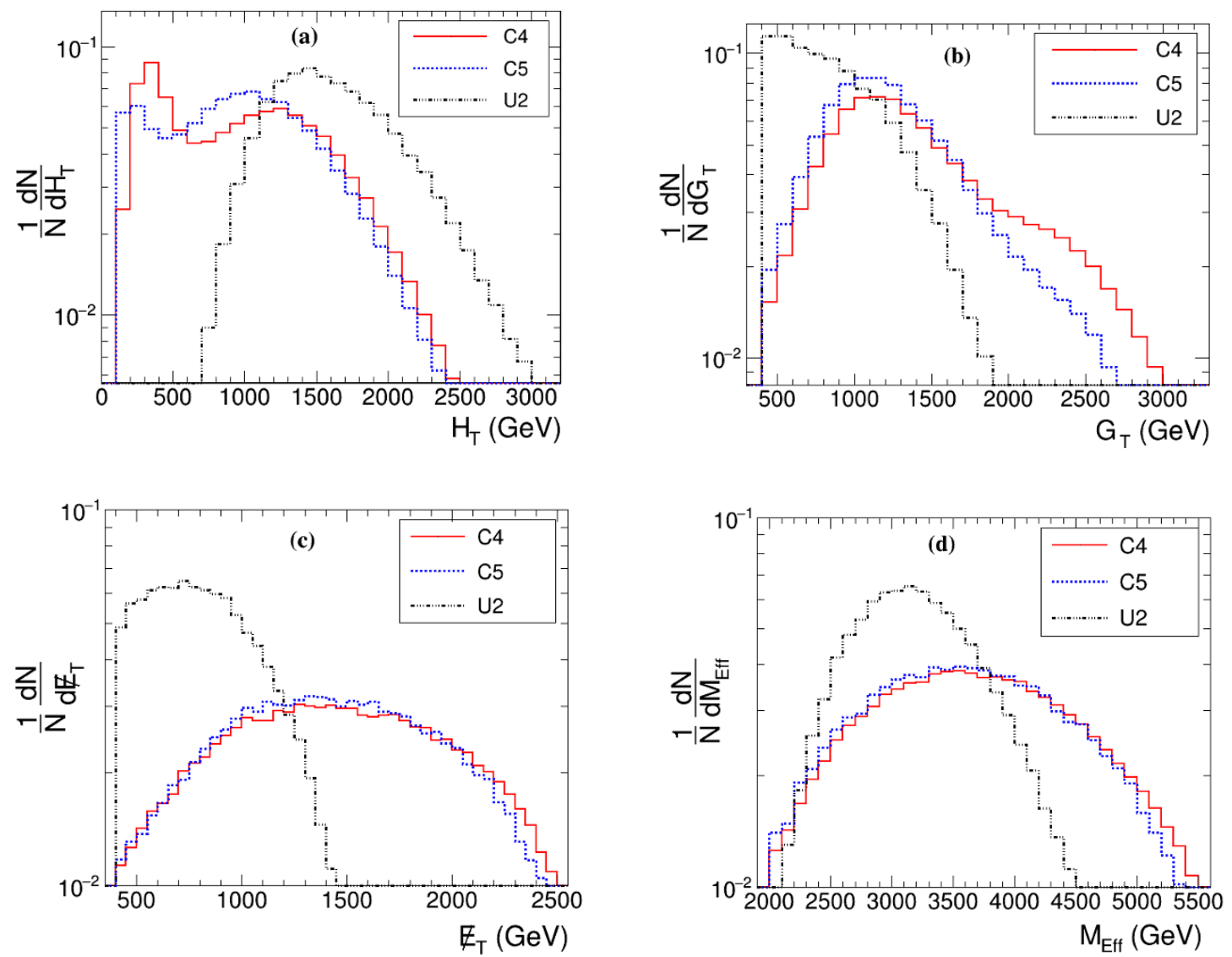

Figure 6. Normalized distributions of total hadronic energy deposit $H_{T}$, total photon energy deposit $G_{T}$, missing transverse energy $\mathbb{F}_{T}$ and Effective Mass $M_{E f f}$, for benchmark points representing various compressed (C4), more compressed (C5) and uncompressed (U2) spectra after implementing the selection and analysis cuts A0-A6.

Taking cue from the kinematic distributions in figure 5 and figure 6 , we now proceed to formulate two observables

$$
r_{1}=\frac{p_{T}\left(j_{1}\right)}{p_{T}\left(\gamma_{1}\right)} \quad \text { and } \quad r_{2}=\frac{p_{T}\left(j_{2}\right)}{p_{T}\left(\gamma_{1}\right)}
$$

which capture the essence of the jet and photon transverse momenta behaviour in a way as to distinctly distinguish between the compressed and uncompressed scenarios. As seen in figure 7 , for the compressed case, $r_{1}$ (figure $7(\mathrm{a})$ ) peaks at rather small values $(\sim 0.1$ ) than the uncompressed case $(\sim 1.0)$ since the leading jet $p_{T}$ is almost always softer than the leading photon for compressed spectra whereas for the uncompressed case there are hard jets with $p_{T}$ values comparable to the leading photon $p_{T}$. However for the compressed spectra, the collimated hard jet from the highly boosted $Z$ boson produced in the decay of the $\widetilde{\chi}_{1}^{0}$, lead to a subdominant peak at $\sim 0.7$ in $r_{1}$. The observable $r_{2}$ (figure $7(\mathrm{~b})$ ) constructed with the sub-leading jet and leading photon $p_{T}$, peaks at lower values $(\sim$ 0.1) for $\mathbf{C} 4$ and $\mathbf{C 5}$ since the sub-leading jet, coming from the cascades or ISR in the 
compressed case is expected to be much softer than the photon. For U2, $r_{2}$ peaks at $\sim 0.5$ since the sub-leading jet also coming from the cascade is softer than the hardest photon. Thus we find that the above ratios seem to enhance the two major distinctive features between a compressed and an uncompressed scenario, namely the high/low $p_{T}$ for the photon/jet for the compressed as compared to the low/high $p_{T}$ of the photon/jet for the uncompressed case.

We further note that the jet multiplicity is another variable which shows a difference in the distributions for compressed spectra C4 and C5 when compared to that of the uncompressed spectra U2 (figure 4(a)). Although the choice of our signal region involves $N_{j}>2$, the compressed spectra, $\mathbf{C 4}$ and $\mathbf{C 5}$, still retain a sufficient fraction of events with higher number of jets. In contrast, the uncompressed spectra U2 has larger number of hard jets for all events, and thereby remains mostly unaffected by this selection criterion. We therefore define a modified ratio (scaled by the jet multiplicities) as

$$
r_{1}^{\prime}=N_{j} r_{1} \quad \text { and } \quad r_{2}^{\prime}=N_{j} r_{2}
$$

Notably the new variables $r_{1}^{\prime}$ and $r_{2}^{\prime}$ are able to significantly enhance the differences between a compressed and uncompressed spectra. Since the scale factor, $N_{j}$, is always greater for the uncompressed spectra U2 than for the compressed spectra $\mathbf{C 4}$ and $\mathbf{C 5}$, we find the peak values of $r_{1}^{\prime}(\sim 4.0)$ and $r_{2}^{\prime}(\sim 2.5)$ of the uncompressed spectra are shifted further away from that of compressed ones $\left(r_{1}^{\prime} \sim 0.2-0.5\right.$ and $\left.r_{2}^{\prime} \sim 0.1-0.3\right)$. Quite importantly the visible overlap seen in $r_{1}$ for the sub-dominant peak is now completely disentangled in the new variable $r_{1}^{\prime}$ as seen in figure $7(\mathrm{c})$. This is significant in the sense that when the event samples would retain a much harder criterion for the leading jet then the events for U2, C4 and C5 would all feature the overlap observed for the sub-dominant peak while the difference for low $r_{1}$ might be washed away for this particular choice of event selection.

Besides enhancing the differences between the compressed and uncompressed spectra, the differential distributions in $r_{i}$ and $r_{i}^{\prime}$ can also be used to highlight the differences amongst the different compressed spectra themselves, depending on the level of compression in mass. For example, $\mathbf{C 4}$, has a larger mass separation $\Delta M_{i}$ than $\mathbf{C 5}$, and shows a peak in the jet multiplicity at $N_{j}=3$ while for C5, the peak value of the differential cross section is at $N_{j}=2$. Thus a larger fraction of events survive after analysis for C4 than C5. Again, since $\mathbf{C 5}$ is relatively more compressed than $\mathbf{C 4}$, the jets from $\mathbf{C 4}$ are considerably harder than the latter. However the NLSP mass for $\mathbf{C 4}$ is larger than $\mathbf{C 5}$, since to probe lower values of compression, we require a heavier NLSP to meet current LHC bounds. This results in the photons being harder for $\mathbf{C 4}$ than for C5. The combined effect of the two seem to be more prominent for both $r_{1}$ and $r_{1}^{\prime}$, where the leading jet is either the ISR jet or cascade jet in case of $\mathbf{C 4}$. For $r_{2}$ this effect seems neutralised, owing to the sub-leading jets for both cases, being much softer than the leading photon $p_{T}$. However the scale factor $N_{j}$ shifts the peak value of $r_{2}^{\prime}$, thus efficiently distinguishing amongst the two compressed spectra of varying degree of compression. 

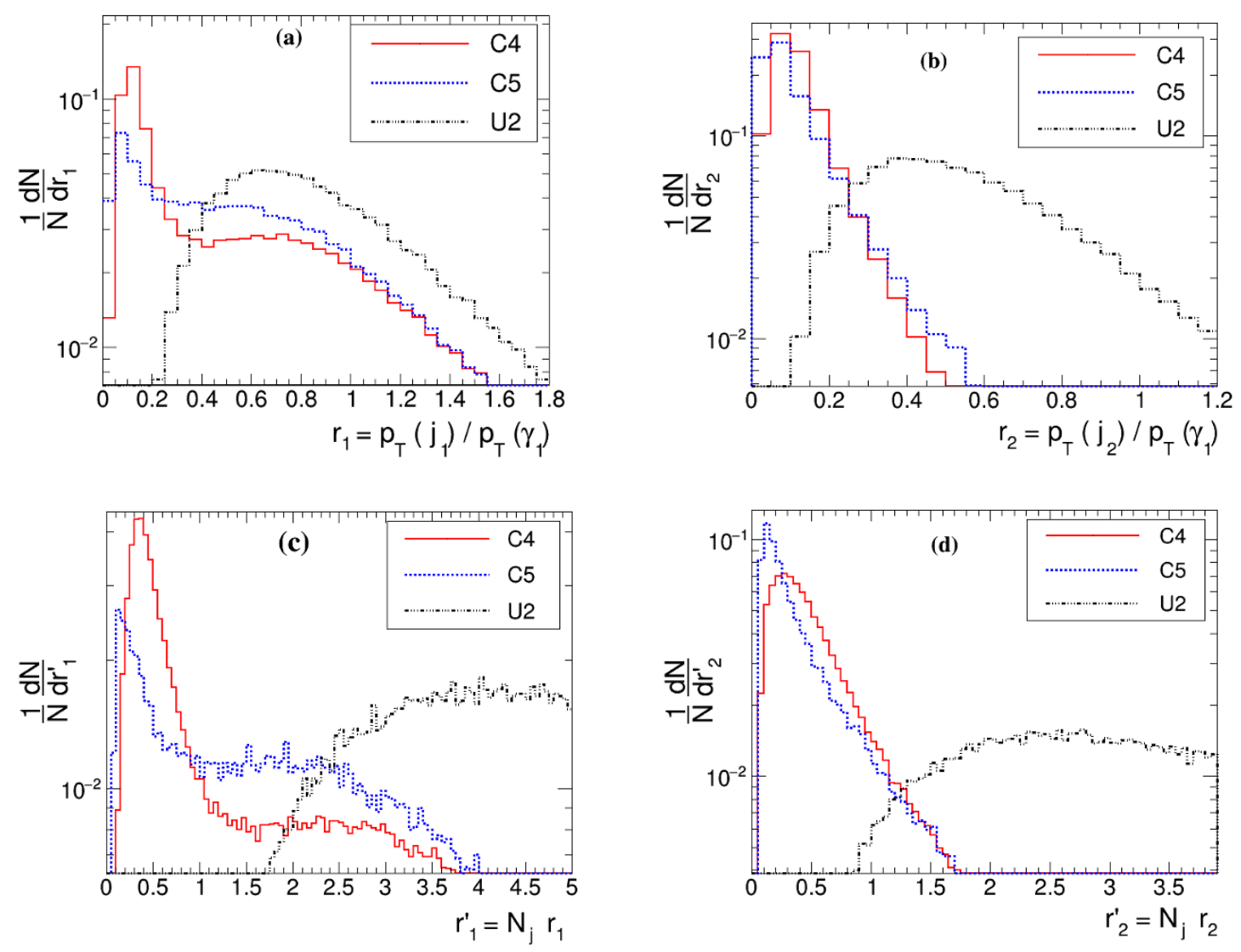

Figure 7. Normalized distributions of different kinematic variables $r_{1}, r_{2}, r_{1}^{\prime}$ and $r_{2}^{\prime}$ to distinguish compressed and uncompressed scenarios for some of the benchmark points representing various compressed (C4), more compressed (C5) and uncompressed (U2) spectra after implementing the selection and analysis cuts A0-A6.

\section{$4.3 \quad$ eV gravitino}

As pointed out earlier that the kinematic characteristics of events when the NLSP decays into a gravitino are independent of whether the $\widetilde{G}$ is in the $\mathrm{keV}$ or eV range. Therefore, for an NLSP decaying into a $\widetilde{G}$ and a SM particle, the $\widetilde{G}$ is practically massless. However, as discussed in section 2.1, a lighter gravitino has a stronger coupling strength to the sparticles. Thus the decay of the sparticles into a SM particle and gravitino dominates over its decay to the NLSP. For a gravitino of mass $1 \mathrm{eV}$, we find that the gluino/squark almost always directly decays to the gravitino rather than to the NLSP. The branching fractions also depend on the mass gap between the coloured sparticles and the NLSP. These features are highlighted in figures 1 and 2 where both compressed and uncompressed mass gaps are shown.

Therefore, an eV $\widetilde{G}$ does affect the overall event rates of the signal in the photon channel when compared to the $\mathrm{keV} \widetilde{G}$ case. An immediate consequence which has gone unnoticed for such light $\mathrm{eV} \widetilde{G}$ case would be a new competing signal which can become 


\begin{tabular}{|c|c|c|c|c|c|c|c|}
\hline \multirow{2}{*}{ Signal } & \multirow{2}{*}{$\begin{array}{c}\text { Production } \\
\text { cross-section (in fb) }\end{array}$} & \multicolumn{5}{|c|}{ Cross-section (in fb) after cuts: } \\
\cline { 3 - 8 } & 0.26 & A0+A1 & A2 & A3 & A4 & A5 & A6 \\
\hline C1 & 0.038 & 0.035 & 0.031 & 0.03 & 0.028 & 0.028 \\
\hline
\end{tabular}

Table 4. Signal Cross-sections (NLO+NLL) for benchmark $\mathbf{C} 1$ for $\geq 1$ photon $+>2$ jets $+\not_{T}$ final states $\left(m_{\widetilde{G}}=1 \mathrm{eV}\right)$.

more relevant than the more popular photonic channel. This can be easily understood by taking a look at the resulting $\operatorname{BR}(\widetilde{g} \rightarrow g \widetilde{G})$ for some of our benchmark points in presence of an $\mathrm{eV}$ gravitino. As indicated by figure 1, this branching ratio is supposed to go up if the spectrum is more compressed. For the same benchmark points as in table 1, now in the presence of an $\mathrm{eV} \widetilde{G}$, we have observed that $\operatorname{BR}(\widetilde{g} \rightarrow g \widetilde{G}) \sim 13 \%, 41 \%$ and $99 \%$ for $\mathbf{U} 1$ $\left(\Delta m_{\widetilde{g} \widetilde{\chi}_{1}^{0}}=1403 \mathrm{GeV}\right), \mathbf{U} \mathbf{2}\left(\Delta m_{\widetilde{g}_{\widetilde{\chi}_{1}^{0}}}=911 \mathrm{GeV}\right)$ and $\mathbf{C 1}\left(\Delta m_{\widetilde{g}_{\widetilde{\chi}_{1}^{0}}}=78 \mathrm{GeV}\right)$ respectively. As a consequence, $\mathbf{C} \mathbf{1}$ with an $\mathrm{eV}$ gravitino, is unlikely to yield a good event rate in the photonic channel since the gluino avoids decaying into the NLSP altogether. However, a small fraction of the squarks may still decay into the NLSP, $\sim 4 \%$ and $\sim 24 \%$ precisely for left and right squarks respectively. Hence, one would still expect a photon signal for such a scenario, but a much weaker one as presented in table 4 .

As expected, the photon signal weakens considerably when compared to one with a keV gravitino and requires an integrated luminosity $\sim 1000 \mathrm{fb}^{-1}$ for observation at the LHC. However, much stronger signal would be obtained in the "n-jet+ $+\mathbb{E}_{T}$ " $(n \geq 2)$ channel as the final state would have at least two very hard ( $p_{T}$ 's exceeding more than $\mathrm{a} \mathrm{TeV}$ ) jets and an equally hard $\not_{T}$ signal for the eV-gravitino case. The conventional multi-jet search [31] rely upon the usual $\mathbb{F}_{T}, M_{E f f}, \frac{\mathbb{F}_{T}}{\sqrt{H_{T}}}$ and $\Delta \phi\left(j, \vec{F}_{T}\right)$ cuts and in some cases, razor variables [32] to reduce the SM backgrounds. We have checked that with these cuts, a $3 \sigma$ significance

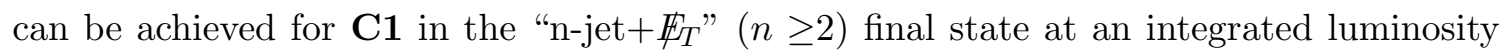
of $\sim 1000 \mathrm{fb}^{-1}$. However, in the presence of an $\mathrm{eV}$ gravitino, one can demand harder $p_{T}$ requirements of the jets and harder $\mathbb{E}_{T}, M_{E f f}$ along with the other conventional cuts to increase signal significance further. We have checked that one can easily bring down the required luminosity to $\sim 728 \mathrm{fb}^{-1}$ for a $3 \sigma$ significance, which is a big improvement over the results obtained for the photon-associated final state. Thus the multi-jet channel is the more favorable one in order to explore an $\mathrm{eV}$ gravitino in presence of a $\sim \mathrm{TeV}$ compressed colour sector. However, as mentioned earlier, such a light gravitino may not be a viable dark matter candidate and would necessarily require the presence of other candidates to satisfy the constraints.

\section{Summary and conclusion}

In this work, we have explored the compressed SUSY scenario in the presence of a light gravitino LSP within the framework of phenomenological MSSM. The question asked is: since the light gravitino produced in the (neutralino) NLSP decays generates as much $\mathbb{E}_{T}$ for compressed spectra as for uncompressed ones, are the former discernible? 
The existing collider studies for such scenarios mostly account for the uncompressed parameter regions, and in some cases the NNLSP-NLSP compressed regions. However, compression in the entire coloured sector of the sparticle spectrum can result in significantly different exclusion limits on the masses of squark, gluino and the lightest neutralino. The presence of a light gravitino in the spectrum affects the branching ratios of the coloured sparticles into $\widetilde{\chi}_{1}^{0}$. We have studied the interplay of these relevant branching ratios for varying $\widetilde{G}$ mass and different amount of compression in the rest of the sparticle spectrum for a bino-like $\widetilde{\chi}_{1}^{0}$. Dictated by the DM constraints, we have mostly concentrated on the keV $\widetilde{G}$ scenario and have performed a detailed collider simulation and cut-based analysis for $\geq 1$ photon $+>2$ jets $+\mathbb{F}_{T}$ final states arising from the squark-gluino pair production channels in the context of the LHC. In our case, the squarks and the gluinos dominantly decay into the $\widetilde{\chi}_{1}^{0}$ which further decay into a $\widetilde{G}$ along with a $\gamma$ or a $Z$ resulting in the above mentioned final state. Hard $p_{T}$ photon requirement can be used along with other kinematic cuts to suppress the SM background very effectively. We have followed the existing ATLAS analysis for the same final state with the help of some benchmark points. We have shown that with the existing experimental data, the exclusion limits on the coloured sparticle masses can increase by $\sim 500 \mathrm{GeV}$ for a highly compressed sparticle spectra. It is understood that similar signal event rates can be obtained from both uncompressed and compressed spectra depending on the choices of masses of squark, gluino and the lightest neutralino. However, the difference in the compression will be reflected in the kinematic distributions of the final state jets and photons. We have exploited this fact to construct some variables which can be used to good effect to differentiate between the two scenarios. We have also studied the collider prospects of SUSY spectra in the presence of sub-keV gravitinos. It turns out that in such cases, the $\widetilde{G}$-associated decay modes of the heavy $(\sim 2.5 \mathrm{TeV})$ coloured sparticles start to become relevant in the presence of high compression between the NNLSP and NLSP. Then the most suitable final state to look for such spectra would be multi-jets $+\mathbb{F}_{T}$. However, the existing DM constraints strongly disfavour presence of such light gravitino in the spectrum.

\section{Acknowledgments}

The work of JD, SM, BM and SKR is partially supported by funding available from the Department of Atomic Energy, Government of India, for the Regional Centre for Acceleratorbased Particle Physics (RECAPP), Harish-Chandra Research Institute, HBNI. PK thanks RECAPP for the hospitality during this work. Computational work for this work was carried out at the cluster computing facility in the Harish-Chandra Research Institute (http://www.hri.res.in/cluster).

Open Access. This article is distributed under the terms of the Creative Commons Attribution License (CC-BY 4.0), which permits any use, distribution and reproduction in any medium, provided the original author(s) and source are credited. 


\section{References}

[1] ATLAS, CMS collaborations, Combined Measurement of the Higgs Boson Mass in pp Collisions at $\sqrt{s}=7$ and 8 TeV with the ATLAS and CMS Experiments, Phys. Rev. Lett. 114 (2015) 191803 [arXiv:1503.07589] [INSPIRE].

[2] ATLAS collaboration, Combined measurements of the Higgs boson production and decay rates in $H \rightarrow Z Z^{*} \rightarrow 4 \ell$ and $H \rightarrow \gamma \gamma$ final states using pp collision data at $\sqrt{s}=13$ TeV in the ATLAS experiment, ATLAS-CONF-2016-081 (2016).

[3] ATLAS collaboration, Observation of a new particle in the search for the Standard Model Higgs boson with the ATLAS detector at the LHC, Phys. Lett. B 716 (2012) 1 [arXiv: 1207.7214] [INSPIRE].

[4] CMS collaboration, Observation of a new boson at a mass of 125 GeV with the CMS experiment at the LHC, Phys. Lett. B 716 (2012) 30 [arXiv:1207.7235] [InSPIRE].

[5] CMS collaboration, Updated measurements of Higgs boson production in the diphoton decay channel at $\sqrt{s}=13$ TeV in pp collisions at CMS., CMS-PAS-HIG-16-020 (2016).

[6] ATLAS collaboration, Measurement of fiducial, differential and production cross sections in the $H \rightarrow \gamma \gamma$ decay channel with $13.3 \mathrm{fb}^{-1}$ of $13 \mathrm{TeV}$ proton-proton collision data with the ATLAS detector, ATLAS-CONF-2016-067 (2016).

[7] ATLAS collaboration, https://atlas.web.cern.ch/Atlas/GROUPS/PHYSICS/CombinedSummaryPlots/SUSY/.

[8] CMS collaboration, https://twiki.cern.ch/twiki/bin/view/CMSPublic/PhysicsResultsSUS.

[9] S.P. Martin, Exploring compressed supersymmetry with same-sign top quarks at the Large Hadron Collider, Phys. Rev. D 78 (2008) 055019 [arXiv:0807.2820] [INSPIRE].

[10] T.J. LeCompte and S.P. Martin, Large Hadron Collider reach for supersymmetric models with compressed mass spectra, Phys. Rev. D 84 (2011) 015004 [arXiv:1105.4304] [INSPIRE].

[11] T.J. LeCompte and S.P. Martin, Compressed supersymmetry after 1/fb at the Large Hadron Collider, Phys. Rev. D 85 (2012) 035023 [arXiv:1111.6897] [InSPIRE].

[12] E. Alvarez and Y. Bai, Reach the Bottom Line of the Sbottom Search, JHEP 08 (2012) 003 [arXiv: 1204.5182] [INSPIRE].

[13] B. Bhattacherjee and K. Ghosh, Degenerate SUSY search at the 8 TeV LHC, arXiv: 1207.6289 [INSPIRE].

[14] G. Bélanger, M. Heikinheimo and V. Sanz, Model-Independent Bounds on Squarks from Monophoton Searches, JHEP 08 (2012) 151 [arXiv:1205.1463] [INSPIRE].

[15] H. Dreiner, M. Krämer and J. Tattersall, Exploring QCD uncertainties when setting limits on compressed supersymmetric spectra, Phys. Rev. D 87 (2013) 035006 [arXiv:1211.4981] [INSPIRE].

[16] B. Bhattacherjee, A. Choudhury, K. Ghosh and S. Poddar, Compressed supersymmetry at 14 TeV LHC, Phys. Rev. D 89 (2014) 037702 [arXiv:1308.1526] [InSPIRE].

[17] S. Mukhopadhyay, M.M. Nojiri and T.T. Yanagida, Compressed SUSY search at the 13 TeV LHC using kinematic correlations and structure of ISR jets, JHEP 10 (2014) 12 [arXiv:1403.6028] [INSPIRE]. 
[18] J. Dutta, P. Konar, S. Mondal, B. Mukhopadhyaya and S.K. Rai, A Revisit to a Compressed Supersymmetric Spectrum with 125 GeV Higgs, JHEP 01 (2016) 051 [arXiv:1511.09284] [INSPIRE].

[19] A. Chakraborty, S. Chakraborty and T.S. Roy, Chasing New Physics in Stacks of Soft Tracks, Phys. Rev. D 94 (2016) 111703 [arXiv:1606.07826] [InSPIRE].

[20] D. Chowdhury, K.M. Patel, X. Tata and S.K. Vempati, Indirect Searches of the Degenerate MSSM, Phys. Rev. D 95 (2017) 075025 [arXiv:1612.06471] [InSPIRE].

[21] P. Nath and A.B. Spisak, Gluino Coannihilation and Observability of Gluinos at LHC RUN II, Phys. Rev. D 93 (2016) 095023 [arXiv: 1603.04854] [INSPIRE].

[22] CMS collaboration, Search for New Physics with a Mono-Jet and Missing Transverse Energy in pp Collisions at $\sqrt{s}=7$ TeV, Phys. Rev. Lett. 107 (2011) 201804 [arXiv:1106.4775] [INSPIRE].

[23] ATLAS collaboration, Search for new phenomena with the monojet and missing transverse momentum signature using the ATLAS detector in $\sqrt{s}=7$ TeV proton-proton collisions, Phys. Lett. B 705 (2011) 294 [arXiv:1106.5327] [InSPIRE].

[24] ATLAS collaboration, Search for squarks and gluinos with the ATLAS detector in final states with jets and missing transverse momentum using $4.7 \mathrm{fb}^{-1}$ of $\sqrt{\mathrm{s}}=7 \mathrm{TeV}$ proton-proton collision data, Phys. Rev. D 87 (2013) 012008 [arXiv:1208.0949] [InSPIRE].

[25] ATLAS collaboration, Search for squarks and gluinos with the ATLAS detector in final states with jets and missing transverse momentum using $\sqrt{s}=8$ TeV proton-proton collision data, JHEP 09 (2014) 176 [arXiv:1405.7875] [INSPIRE].

[26] CMS collaboration, Search for new physics in the multijet and missing transverse momentum final state in proton-proton collisions at $\sqrt{s}=8 \mathrm{TeV}$, JHEP 06 (2014) 055 [arXiv: 1402.4770] [INSPIRE].

[27] CMS collaboration, Search for dark matter, extra dimensions and unparticles in monojet events in proton-proton collisions at $\sqrt{s}=8 \mathrm{TeV}$, Eur. Phys. J. C $7 \mathbf{5}(2015) 235$ [arXiv: 1408.3583] [INSPIRE].

[28] ATLAS collaboration, Search for new phenomena in final states with an energetic jet and large missing transverse momentum in pp collisions at $\sqrt{s}=13$ TeV using the ATLAS detector, Phys. Rev. D 94 (2016) 032005 [arXiv: 1604.07773] [INSPIRE].

[29] ATLAS collaboration, Search for new phenomena in final states with an energetic jet and large missing transverse momentum in pp collisions at $\sqrt{s}=8 \mathrm{TeV}$ with the ATLAS detector, Eur. Phys. J. C 75 (2015) 299 [arXiv:1502.01518] [INSPIRE].

[30] ATLAS collaboration, Search for squarks and gluinos in final states with jets and missing transverse momentum at $\sqrt{s}=13 \mathrm{TeV}$ with the ATLAS detector, Eur. Phys. J. C 76 (2016) 392 [arXiv: 1605.03814] [INSPIRE].

[31] ATLAS collaboration, Further searches for squarks and gluinos in final states with jets and missing transverse momentum at $\sqrt{s}=13 \mathrm{TeV}$ with the ATLAS detector, ATLAS-CONF-2016-078 (2016).

[32] CMS Collaboration, An inclusive search for new phenomena in final states with one or more jets and missing transverse momentum at $13 \mathrm{TeV}$ with the AlphaT variable, CMS-SUS-16-016 (2016). 
[33] S. Dimopoulos, M. Dine, S. Raby and S.D. Thomas, Experimental signatures of low-energy gauge mediated supersymmetry breaking, Phys. Rev. Lett. 76 (1996) 3494 [hep-ph/9601367] [INSPIRE].

[34] F. Zwirner, Effective interactions of a light gravitino, Fortsch. Phys. 47 (1999) 325 [hep-th/9801003] [INSPIRE].

[35] G.F. Giudice and R. Rattazzi, Theories with gauge mediated supersymmetry breaking, Phys. Rept. 322 (1999) 419 [hep-ph/9801271] [INSPIRE].

[36] J.L. Feng, M. Kamionkowski and S.K. Lee, Light Gravitinos at Colliders and Implications for Cosmology, Phys. Rev. D 82 (2010) 015012 [arXiv: 1004.4213] [INSPIRE].

[37] J.T. Ruderman and D. Shih, General Neutralino NLSPs at the Early LHC, JHEP 08 (2012) 159 [arXiv: 1103.6083] [INSPIRE].

[38] J.S. Kim, M.E. Krauss and V. Martin-Lozano, Probing the Electroweakino Sector of General Gauge Mediation at the LHC, arXiv:1705.06497 [InSPIRE].

[39] H.K. Dreiner, M. Hanussek, J.S. Kim and S. Sarkar, Gravitino cosmology with a very light neutralino, Phys. Rev. D 85 (2012) 065027 [arXiv:1111.5715] [INSPIRE].

[40] B.C. Allanach, M. Badziak, G. Cottin, N. Desai, C. Hugonie and R. Ziegler, Prompt Signals and Displaced Vertices in Sparticle Searches for Next-to-Minimal Gauge Mediated Supersymmetric Models, Eur. Phys. J. C 76 (2016) 482 [arXiv:1606.03099] [InSPIRE].

[41] ATLAS collaboration, Search for diphoton events with large missing transverse momentum in $7 \mathrm{TeV}$ proton-proton collision data with the ATLAS detector, Phys. Lett. B 718 (2012) 411 [arXiv: 1209.0753] [INSPIRE].

[42] ATLAS collaboration, Search for photonic signatures of gauge-mediated supersymmetry in $8 \mathrm{TeV}$ pp collisions with the ATLAS detector, Phys. Rev. D 92 (2015) 072001 [arXiv: 1507.05493] [INSPIRE].

[43] CMS Collaboration, Search for supersymmetry in events with photons and missing transverse energy, CMS-PAS-SUS-15-012 (2016).

[44] CMS collaboration, Search for supersymmetry in electroweak production with photons and large missing transverse energy in pp collisions at $\sqrt{s}=8$ TeV, Phys. Lett. B 759 (2016) 479 [arXiv: 1602.08772] [INSPIRE].

[45] CMS Collaboration, Search for supersymmetry in final states with at least one photon and MET in pp collisions at $\sqrt{s}=13$ TeV, CMS-PAS-SUS-16-023 (2016).

[46] ATLAS collaboration, Search for supersymmetry in a final state containing two photons and missing transverse momentum in $\sqrt{s}=13 \mathrm{TeV}$ pp collisions at the LHC using the ATLAS detector, Eur. Phys. J. C 76 (2016) 517 [arXiv:1606.09150] [INSPIRE].

[47] CMS collaboration, Search for supersymmetry in events with photons and missing transverse energy in pp collisions at 13 TeV, Phys. Lett. B 769 (2017) 391 [arXiv:1611.06604] [INSPIRE].

[48] ATLAS collaboration, Search for Supersymmetry in events with photons, jets and missing transverse energy with the ATLAS detector in $13 \mathrm{TeV}$ pp collisions, ATLAS-CONF-2016-066 (2016).

[49] A. Arbey, M. Battaglia, A. Djouadi, F. Mahmoudi and J. Quevillon, Implications of a 125 GeV Higgs for supersymmetric models, Phys. Lett. B 708 (2012) 162 [arXiv:1112.3028] [INSPIRE]. 
[50] M.A. Ajaib, I. Gogoladze, F. Nasir and Q. Shafi, Revisiting mGMSB in Light of a $125 \mathrm{GeV}$ Higgs, Phys. Lett. B $\mathbf{7 1 3}$ (2012) 462 [arXiv:1204.2856] [INSPIRE].

[51] A. Djouadi, Implications of the Higgs discovery for SUSY, J. Phys. Conf. Ser. 447 (2013) 012002 [INSPIRE].

[52] A. Albaid and K.S. Babu, Higgs boson of mass $125 \mathrm{GeV}$ in GMSB models with messenger-matter mixing, Phys. Rev. D 88 (2013) 055007 [arXiv:1207.1014] [INSPIRE].

[53] P. Byakti and T.S. Ray, Burgeoning the Higgs mass to $125 \mathrm{GeV}$ through messenger-matter interactions in GMSB models, JHEP 05 (2013) 055 [arXiv:1301.7605] [INSPIRE].

[54] E.A. Baltz and H. Murayama, Gravitino warm dark matter with entropy production, JHEP 05 (2003) 067 [astro-ph/0108172] [INSPIRE].

[55] M. Viel, J. Lesgourgues, M.G. Haehnelt, S. Matarrese and A. Riotto, Constraining warm dark matter candidates including sterile neutrinos and light gravitinos with WMAP and the Lyman-alpha forest, Phys. Rev. D 71 (2005) 063534 [astro-ph/0501562] [INSPIRE].

[56] L. Covi, J. Hasenkamp, S. Pokorski and J. Roberts, Gravitino Dark Matter and general neutralino NLSP, JHEP 11 (2009) 003 [arXiv:0908.3399] [INSPIRE].

[57] L. Covi, M. Olechowski, S. Pokorski, K. Turzynski and J.D. Wells, Supersymmetric mass spectra for gravitino dark matter with a high reheating temperature, JHEP 01 (2011) 033 [arXiv: 1009.3801] [INSPIRE].

[58] A. Arbey, M. Battaglia, L. Covi, J. Hasenkamp and F. Mahmoudi, LHC constraints on Gravitino Dark Matter, Phys. Rev. D 92 (2015) 115008 [arXiv:1505.04595] [InSPIRE].

[59] L. Covi, Dark matter candidates: axino and gravitino, in proceedings of the 46th Rencontres de Moriond on Electroweak Interactions and Unified Theories, La Thuile, Italy, 13-20 March 2011, pp. 381-388.

[60] A. Boyarsky, J. Lesgourgues, O. Ruchayskiy and M. Viel, Lyman-alpha constraints on warm and on warm-plus-cold dark matter models, JCAP 05 (2009) 012 [arXiv:0812.0010] [INSPIRE].

[61] J. Baur, N. Palanque-Delabrouille, C. Yèche, C. Magneville and M. Viel, Lyman-alpha Forests cool Warm Dark Matter, JCAP 08 (2016) 012 [arXiv:1512.01981] [INSPIRE].

[62] M. Drees, R. Godbole and P. Roy, Theory and phenomenology of sparticles: an account of four-dimensional $N=1$ supersymmetry in high energy physics, World Scientific Publishing (2004).

[63] W. Porod, The Decays gluino $\rightarrow$ stop (1) $\bar{b} W$ and gluino $\rightarrow$ stop (1) $\bar{c}$ and phenomenological implications in supersymmetric theories, JHEP 05 (2002) 030 [hep-ph/0202259] [INSPIRE].

[64] W. Porod, SPheno, a program for calculating supersymmetric spectra, SUSY particle decays and SUSY particle production at $e^{+} e^{-}$colliders, Comput. Phys. Commun. 153 (2003) 275 [hep-ph/0301101] [INSPIRE].

[65] W. Porod and F. Staub, SPheno 3.1: Extensions including flavour, CP-phases and models beyond the MSSM, Comput. Phys. Commun. 183 (2012) 2458 [arXiv:1104.1573] [INSPIRE].

[66] J. Alwall, M. Herquet, F. Maltoni, O. Mattelaer and T. Stelzer, MadGraph 5: Going Beyond, JHEP 06 (2011) 128 [arXiv:1106.0522] [INSPIRE].

[67] J. Alwall, R. Frederix, S. Frixione, V. Hirschi, F. Maltoni, O. Mattelaer et al., The automated computation of tree-level and next-to-leading order differential cross sections and their matching to parton shower simulations, JHEP 07 (2014) 079 [arXiv:1405.0301] [INSPIRE]. 
[68] T. Sjöstrand, S. Mrenna and P.Z. Skands, PYTHIA 6.4 Physics and Manual, JHEP 05 (2006) 026 [hep-ph/0603175] [INSPIRE].

[69] M.L. Mangano, M. Moretti, F. Piccinini and M. Treccani, Matching matrix elements and shower evolution for top-quark production in hadronic collisions, JHEP 01 (2007) 013 [hep-ph/0611129] [INSPIRE].

[70] S. Hoeche, F. Krauss, N. Lavesson, L. Lönnblad, M. Mangano, A. Schalicke et al., Matching parton showers and matrix elements, in proceedings of the HERA and the LHC: a Workshop on the implications of HERA for LHC physics, part A, 2006 [hep-ph/0602031] [INSPIRE].

[71] https://cp3.irmp.ucl.ac.be/projects/madgraph/wiki/IntroMatching.

[72] https://cp3.irmp.ucl.ac.be/projects/madgraph/wiki/FAQ-General-13.

[73] J. Pumplin, D.R. Stump, J. Huston, H.L. Lai, P.M. Nadolsky and W.K. Tung, New generation of parton distributions with uncertainties from global QCD analysis, JHEP 07 (2002) 012 [hep-ph/0201195] [INSPIRE].

[74] DELPHES 3 collaboration, J. de Favereau, C. Delaere, P. Demin, A. Giammanco, V. Lemaitre, A. Mertens et al., DELPHES 3, A modular framework for fast simulation of a generic collider experiment, JHEP 02 (2014) 057 [arXiv: 1307.6346] [INSPIRE].

[75] M. Selvaggi, DELPHES 3: A modular framework for fast-simulation of generic collider experiments, J. Phys. Conf. Ser. 523 (2014) 012033 [INSPIRE].

[76] A. Mertens, New features in Delphes 3, J. Phys. Conf. Ser. 608 (2015) 012045 [inSPIRE].

[77] M. Cacciari, G.P. Salam and G. Soyez, FastJet User Manual, Eur. Phys. J. C 72 (2012) 1896 [arXiv: 1111.6097] [INSPIRE].

[78] M. Cacciari, G.P. Salam and G. Soyez, The Anti-k(t) jet clustering algorithm, JHEP 04 (2008) 063 [arXiv:0802.1189] [INSPIRE].

[79] W. Beenakker, R. Hopker, M. Spira and P.M. Zerwas, Squark and gluino production at hadron colliders, Nucl. Phys. B 492 (1997) 51 [hep-ph/9610490] [InSPIRE].

[80] A. Kulesza and L. Motyka, Threshold resummation for squark-antisquark and gluino-pair production at the LHC, Phys. Rev. Lett. 102 (2009) 111802 [arXiv:0807.2405] [INSPIRE].

[81] A. Kulesza and L. Motyka, Soft gluon resummation for the production of gluino-gluino and squark-antisquark pairs at the LHC, Phys. Rev. D 80 (2009) 095004 [arXiv: 0905.4749] [INSPIRE].

[82] W. Beenakker, S. Brensing, M. Krämer, A. Kulesza, E. Laenen and I. Niessen, Soft-gluon resummation for squark and gluino hadroproduction, JHEP 12 (2009) 041 [arXiv: 0909.4418] [INSPIRE].

[83] W. Beenakker, C. Borschensky, M. Krämer, A. Kulesza, E. Laenen, S. Marzani et al., $N L O+N L L$ squark and gluino production cross-sections with threshold-improved parton distributions, Eur. Phys. J. C 76 (2016) 53 [arXiv:1510.00375] [INSPIRE]. 